TRANSACTIONS OF THE

AMERICAN MATHEMATICAL SOCIETY

Volume 362, Number 4, April 2010, Pages 1797-1830

S 0002-9947(09)04634-0

Article electronically published on October 26, 2009

\title{
DECAY RATES OF STRONG PLANAR RAREFACTION WAVES \\ TO SCALAR CONSERVATION LAWS WITH DEGENERATE VISCOSITY IN SEVERAL SPACE DIMENSIONS
}

\author{
JING CHEN AND CHANGJIANG ZHU
}

\begin{abstract}
This paper is concerned with the decay rates of the solution to the strong planar rarefaction waves for scalar conservation laws with degenerate viscosity in several space dimensions. The analysis is based on the $L^{2}$-energy method and the decay property of rarefaction waves.
\end{abstract}

\section{INTRODUCTION}

In this paper, we will investigate the decay rates of the solution to the strong planar rarefaction waves for scalar conservation laws with degenerate viscosity, which is called the 'generalized Burgers equation', in two or more space dimensions. Since the proof of the result for the case in more than two dimensions is identical to that for the case in two dimensions, we only discuss the equation of the following form in two-dimensional space:

$$
u_{t}+f(u)_{x}+g(u)_{y}=t^{\alpha}\left(u_{x x}+u_{y y}\right), \quad(t, x, y) \in \mathbb{R}^{+} \times \mathbb{R}^{2},
$$

where $\alpha$ is a positive constant, $f$ and $g$ are smooth functions (say in $C^{3}$ ). Furthermore, we assume that $f$ is convex, i.e., there exists a positive constant $\beta$ such that

$$
f^{\prime \prime}(u) \geq \beta \quad \text { for } u \in \mathbb{R} .
$$

The initial data for equation (1.1) is

$$
u(0, x, y)=u_{0}(x, y)
$$

satisfying

$$
\lim _{x \rightarrow \pm \infty}\left\|u_{0}(x, \cdot)-u_{ \pm}\right\|_{L^{\infty}(\mathbb{R})}=0,
$$

where $u_{ \pm}$are two constants with $u_{-}<u_{+}$.

Equation (1.1) is not only a mathematical model of the propagation of the finiteamplitude sound waves of a duct with variable area, where $u$ is an acoustic variable (see [1, 2]), but it can describe the unsteady Navier-Stokes system with cylindrical symmetry motion in small amplitude (see [8, 12]).

Considering that it has a wide physical background, many authors have shown great interest in studying the properties; cf. [1, 2, 13, 14, 16, 17.

Received by the editors July 28, 2005 and, in revised form, August 1, 2007.

2000 Mathematics Subject Classification. Primary 35L65, 35K65, 35B40, 35B45.

Key words and phrases. Strong planar rarefaction waves, energy method, a priori estimates, decay rates.

(C)2009 American Mathematical Society Reverts to public domain 28 years from publication 
When $\alpha=0$, many papers have discussed the existence and asymptotic behavior of the solutions to the corresponding Cauchy problem (1.1)-(1.4). For the case of one space dimension, first, in 1960, Il'in and Oleinik originally investigated the asymptotic behavior of solutions to the Cauchy problem for a generalized Burgers equation of the following form (see [5]):

$$
\begin{cases}u_{t}+f(u)_{x}=\mu u_{x x}, & \mu>0, \quad(t, x) \in \mathbb{R}^{+} \times \mathbb{R} \\ u(0, x)=u_{0}(x) \rightarrow u_{ \pm}, & x \rightarrow \pm \infty\end{cases}
$$

Then, Harabetian obtained the convergence rate toward the rarefaction wave (see [3]). Furthermore, Hattori and Nishihara showed more precise behavior of the solutions for the Burgers equation, employing the Hopf-Cole transformation (see [4]). For the case of several space dimensions, Xin first studied the stability of the weak planar rarefaction wave for the scalar conservation laws with viscosity (see [15]). Ito showed the convergence rate toward the weak planar rarefaction wave (see [6]). Recently, Nishikawa and Nishihara in [1] obtained the decay rate toward the strong planar rarefaction wave, and Zhao in [18] also obtained nonlinear stability of the strong planar rarefaction wave for a relaxation model in several space dimensions.

When $\alpha>0$, the global existence of smooth solutions of problem (1.1), (1.3) in one space dimension has been obtained for $\alpha=1$ and $f(u)=\frac{1}{2} u^{2}$ (see [1, 2, 13, 14, 17]). Recently, Xu and Jiang proved that, when $\alpha \in\left[0, \frac{1}{4 q}\right)$ with $q>\frac{3}{2}$, there exist global smooth solutions of the Cauchy problem (1.1)-(1.4) in one space dimension and showed the corresponding solutions converge asymptotically to the weak rarefaction wave (see [16]).

Our main purpose of this paper is to show when $\alpha \in\left(0, \frac{1}{7}\right)$, the Cauchy problem (1.1)-(1.4) admits a unique global smooth solution, and this solution converges asymptotically to the strong planar rarefaction wave. Moreover, the corresponding decay rates are also obtained by energy methods and the decay property of rarefaction waves. However, in this paper, we are forced to use more complex skills because of the difficulty caused by the degenerate singularity at $t=0$ and at $t=\infty$. To do this, we separate our estimates into two parts. For one part, we use a Gronwall inequality to overcome the degenerate singularity at $t=0$. For the other, we make use of the decay property of the rarefaction wave to deal with the singularity at $t=\infty$.

According to the idea of asymptotic analysis, it is natural to expect that the solution is closely related to that of the Riemann problem for the corresponding scalar hyperbolic conservation law in one space dimension:

$$
\begin{array}{r}
u_{t}+f(u)_{x}=0, \quad(t, x) \in \mathbb{R}^{+} \times \mathbb{R}, \\
u(0, x)=u_{0}^{R}(x)= \begin{cases}u_{-}, & x<0, \\
u_{+}, & x>0 .\end{cases}
\end{array}
$$

The entropy solution $u^{R}(t, x)$ of $(1.5),(1.6)$, which is called the central rarefaction wave, is given by

$$
u^{R}(t, x)=\left\{\begin{array}{lll}
u_{-}, & \text {for } & x \leq f^{\prime}\left(u_{-}\right) t, \\
\left(f^{\prime}\right)^{-1}\left(\frac{x}{t}\right), & \text { for } \quad & f^{\prime}\left(u_{-}\right) t<x<f^{\prime}\left(u_{+}\right) t, \\
u_{+}, & \text {for } \quad x \geq f^{\prime}\left(u_{+}\right) t .
\end{array}\right.
$$


Notation. Throughout this paper, we denote positive constants by $C$. Moreover, the character " $C$ " may differ in different places. $L^{p}=L^{p}(\mathbb{R})$ (or $L^{p}\left(\mathbb{R}^{2}\right)$ ) $(1 \leq p \leq \infty)$ denotes the usual Lebesgue space on $\mathbb{R}$ (or $\mathbb{R}^{2}$ ) with its norm $\|f\|_{L^{p}}=\left(\int_{\mathbb{R}}|f(x)|^{p} d x\right)^{\frac{1}{p}}\left(\right.$ or $\left.\left(\int_{\mathbb{R}^{2}}|f(x, y)|^{p} d x d y\right)^{\frac{1}{p}}\right), \quad 1 \leq p<\infty,\|f\|_{L^{\infty}}=$ $\sup _{\mathbb{R}}|f(x)|\left(\right.$ or $\left.\sup _{\mathbb{R}^{2}}|f(x, y)|\right)$, and when $p=2$, we write $\|\cdot\|_{L^{2}}=\|\cdot\| . H^{l}=H^{l}(\mathbb{R})$ (or $H^{l}\left(\mathbb{R}^{2}\right)$ ) denotes the usual $l$ th-order Sobolev space with its norm $\|f\|_{H^{l}}=$ $\|f\|_{l}=\left(\sum_{i=0}^{l}\left\|\partial_{x}^{i} f\right\|^{2}\right)^{\frac{1}{2}}$. For simplicity, $\|f(\cdot, t)\|_{L^{p}}$ and $\|f(\cdot, t)\|_{l}$ are denoted by $\|f(t)\|_{L^{p}}$ and $\|f(t)\|_{l}$, respectively.

Denote

$$
\mathbb{R}_{+}^{2}=\left\{(x, y) \in \mathbb{R}^{2}, \quad x>0\right\}, \quad \mathbb{R}_{-}^{2}=\left\{(x, y) \in \mathbb{R}^{2}, \quad x<0\right\},
$$

and

$$
D=\left(\frac{\partial}{\partial x}, \frac{\partial}{\partial y}\right) .
$$

Then our main theorem is stated as follows:

Theorem 1.1 (Main result). Suppose that $u_{0}-u_{ \pm} \in L^{2}\left(\mathbb{R}_{ \pm}^{2}\right) \cap L^{1}\left(\mathbb{R}_{ \pm}^{2}\right)$ and $D^{r} u_{0} \in H^{1}$ with $|r|=1$. Then, for any $0<\alpha<\frac{1}{7}$, the Cauchy problem (1.1)-(1.4) admits a unique global smooth solution $u(t, x, y)$ satisfying

$$
\sup _{y \in \mathbb{R}}\left\|u(t, \cdot, y)-u^{R}(t, \cdot)\right\|_{L^{2}\left(\mathbb{R}_{x}\right)} \leq C(1+t)^{-\frac{1}{4}+\frac{3 \alpha}{4}},
$$

where $C$ is a positive constant depending on $u_{0}, u_{-}$and $u_{+}$, but independent of $t$.

The plan of the paper is as follows. In Section 2, we construct a smooth approximate rarefaction wave to reformulate our problem. In Sections 3 and 4, we will solve the two reformulated problems by the energy method.

\section{Reformulation of the PRoblem}

In this section, we will construct a smooth approximate rarefaction wave and reformulate our problem. Then we will introduce several useful lemmas which will be used frequently later.

Consider the inviscid Burgers equation

$$
w_{t}^{R}+w^{R} w_{x}^{R}=0,
$$

with Riemann initial data

$$
w^{R}(0, x)=w_{0}^{R}(x)= \begin{cases}w_{-}, & x<0, \\ w_{+}, & x>0,\end{cases}
$$

where $w_{ \pm}=f^{\prime}\left(u_{ \pm}\right)$with $w_{-}<w_{+}$.

It is well known that the Riemann problem (2.1) and (2.2) has a central rarefaction wave $w^{R}(t, x)=w^{R}\left(\frac{x}{t}\right)$, where

$$
w^{R}\left(\frac{x}{t}\right)= \begin{cases}w_{-}, & x \leq w_{-} t, \\ \frac{x}{t}, & w_{-} t<x<w_{+} t, \\ w_{+}, & x \geq w_{+} t .\end{cases}
$$


Since $w^{R}(t, x)$ is not smooth, we construct a smooth approximate rarefaction wave $W(t, x)$ as follows (see [9, 10, 19]):

$$
W_{t}+W W_{x}=0
$$

with smooth initial data

$$
W(0, x)=W_{0}(x)=\frac{1}{2}\left(w_{+}+w_{-}\right)+\frac{1}{2}\left(w_{+}-w_{-}\right) K \int_{0}^{\left(u_{+}-u_{-}\right) x}\left(1+y^{2}\right)^{-1} d y,
$$

where $K$ is a constant satisfying

$$
K \int_{0}^{+\infty}\left(1+y^{2}\right)^{-1} d y=1 .
$$

Now let

$$
w(t, x)=\left(f^{\prime}\right)^{-1}(W(t, x))
$$

Then we have

$$
\left\{\begin{array}{l}
w_{t}+f(w)_{x}=0 \\
w(0, x)=w_{0}(x)=\left(f^{\prime}\right)^{-1}\left(W_{0}(x)\right) \rightarrow u_{ \pm}, \quad \text { as } \quad x \rightarrow \pm \infty .
\end{array}\right.
$$

For convenience, we list the following results on the smooth approximate rarefaction wave $w(t, x)$ which will be used later.

Lemma 2.1. The smooth approximate rarefaction wave $w(t, x)$ satisfies:

$$
u_{-}<w(t, x)<u_{+}, \quad w_{x}(t, x)>0 \text { for any }(t, x) \in \mathbb{R}^{+} \times \mathbb{R} .
$$

(ii) For any $1 \leq p \leq \infty$, there exists a constant $C_{p}$ depending on $p$, such that

$$
\begin{gathered}
\left\|w_{x}(t)\right\|_{L^{p}}^{p} \leq C_{p}(1+t)^{-p+1}, \\
\left\|w_{x x}(t)\right\|_{L^{p}}^{p} \leq C_{p}(1+t)^{-\frac{3 p-1}{2}}, \\
\left\|w_{x x x}(t)\right\|_{L^{p}}^{p} \leq C_{p}(1+t)^{-2 p+1}, \\
\left\|w(t, \cdot)-u^{R}(t, \cdot)\right\|_{L^{p}}^{p} \leq C t^{-\frac{p-1}{2}} .
\end{gathered}
$$

The proofs of properties (2.7)-(2.10) can be found in [9, 10, 19. As for the estimate (2.11), we can obtain it by a similar method as used in [6].

In order to reformulate our problems, we introduce a diffusion wave $U(t, x)$ to approximate $w(t, x)$ and which satisfies

$$
\left\{\begin{array}{l}
U_{t}+f(U)_{x}=t^{\alpha} U_{x x} \\
U(0, x)=U_{0}(x) \rightarrow u_{ \pm}, \quad \text { as } \quad x \rightarrow \pm \infty
\end{array}\right.
$$

The unique global smooth solution of (2.12) was obtained in [16].

By the same argument as those in [15], we can prove the following lemma:

Lemma 2.2. Suppose that $U_{0}(x)$ is monotonically increasing; i.e., $\frac{d}{d x} U_{0}(x)>0$ for any $x \in \mathbb{R}$. Then $U(t, x)$ satisfies

$$
\frac{\partial}{\partial x} U(t, x)>0, \quad(t, x) \in \mathbb{R}^{+} \times \mathbb{R} .
$$

Let

$$
v(t, x)=U(t, x)-w(t, x), \quad V(t, x, y)=u(t, x, y)-U(t, x),
$$

where $w(t, x)$ and $U(t, x)$ are defined by (2.5) and (2.12), respectively. 
Then

$$
u(t, x, y)-u^{R}(t, x)=V(t, x, y)+v(t, x)+\left\{w(t, x)-u^{R}(t, x)\right\} .
$$

Therefore, in order to prove Theorem 1.1, from the estimate (2.11), we need only to estimate $v(t, x)$ and $V(t, x, y)$.

By (2.12) and (1.1), it is easy to verify that the $v(t, x)$ and $V(t, x, y)$ satisfy the following two reformulated problems:

$$
v_{t}+(f(v+w)-f(w))_{x}=t^{\alpha} v_{x x}+t^{\alpha} w_{x x}, \quad(t, x) \in \mathbb{R}^{+} \times \mathbb{R},
$$

with initial data

$$
v(0, x)=U_{0}(x)-w_{0}(x) \equiv v_{0}(x),
$$

and

with initial data

$$
V(0, x, y)=u_{0}(x, y)-U_{0}(x) \equiv V_{0}(x, y) .
$$

In the next two sections, we will devote ourselves to obtaining the decay rates of $v(t, x)$ and $V(t, x, y)$, respectively.

\section{Decay estimates for the Perturbation $v(t, x)$}

For any $0<T \leq \infty$, define

$$
X_{1}(0, T)=\left\{v(t, x): v \in L^{\infty}\left([0, T] ; H^{2}\right), t^{\frac{\alpha}{2}} v_{x} \in L^{2}\left([0, T] ; H^{2}\right)\right\} .
$$

Suppose that $v_{0} \in H^{2}$. By a standard method (see [14, 16, 20]), we can obtain $v(t, x) \in X_{1}\left(0, t_{0}\right)$, where $t_{0}$ depends on $\left\|v_{0}\right\|_{2}$.

Therefore, in order to get the global existence of solutions to (2.14) and (2.15) in $X_{1}(0, \infty)$, it is sufficient to get a priori estimates in $X_{1}(0, \infty)$. To do this, we need to establish the $L^{1}$-estimate on $v(t, x)$.

Lemma 3.1 ( $L^{1}$-estimate). Suppose that $v_{0} \in L^{1} \cap H^{2}$. Then the solution $v(t, x)$ of (2.14) and (2.15) satisfies

$$
\|v(t, \cdot)\|_{L^{1}} \leq\left\|v_{0}\right\|_{L^{1}}+C(1+t)^{\alpha} .
$$

Proof. According to the idea in [6], let $j_{\delta}(\lambda)$ be the usual smoothing kernel in $\mathbb{R}^{1}$; i.e.,

$$
j_{\delta}(\lambda)=\delta^{-1} j\left(\frac{\lambda}{\delta}\right),
$$

where $j(\lambda)$ is a smooth function which has a compact support and satisfies $\int_{\mathbb{R}} j(\lambda) d \lambda$ $=1$. Let $\phi_{\delta}$ be the convolution of the sign function and $j_{\delta}$, i.e.,

$$
\phi_{\delta}(\lambda)=\left(j_{\delta} * \operatorname{sign}\right)(\lambda),
$$

and put

$$
\Phi_{\delta}(\lambda)=\int_{0}^{\lambda} \phi_{\delta}(\xi) d \xi
$$


Multiplying (2.14) by $\phi_{\delta}(v)$ and integrating it with respect to $t$ and $x$, we have

$$
\begin{aligned}
\int_{\mathbb{R}} \Phi_{\delta}(v) d x+\int_{0}^{t} \int_{\mathbb{R}} \phi_{\delta}(v)(f(v+w)-f(w))_{x} d x d \tau \\
=\int_{\mathbb{R}} \Phi_{\delta}\left(v_{0}\right) d x+\int_{0}^{t} \int_{\mathbb{R}} \tau^{\alpha} \phi_{\delta}(v) v_{x x} d x d \tau+\int_{0}^{t} \int_{\mathbb{R}} \tau^{\alpha} \phi_{\delta}(v) w_{x x} d x d \tau .
\end{aligned}
$$

Similar to [6], we can obtain

$$
\begin{gathered}
\int_{\mathbb{R}} \Phi_{\delta}(v) d x \rightarrow \int_{\mathbb{R}}|v| d x, \quad \text { as } \quad \delta \rightarrow 0, \\
\int_{\mathbb{R}} \Phi_{\delta}\left(v_{0}\right) d x \rightarrow \int_{\mathbb{R}}\left|v_{0}\right| d x, \quad \text { as } \quad \delta \rightarrow 0, \\
\int_{0}^{t} \int_{\mathbb{R}} \phi_{\delta}(v)(f(v+w)-f(w))_{x} d x d \tau \rightarrow 0, \quad \text { as } \quad \delta \rightarrow 0 \text { for each } t \in \mathbb{R}^{+},
\end{gathered}
$$

and

$$
\int_{0}^{t} \int_{\mathbb{R}} \tau^{\alpha} \phi_{\delta}(v) v_{x x} d x d \tau=-\int_{0}^{t} \int_{\mathbb{R}} \tau^{\alpha} \phi_{\delta}^{\prime}(v) v_{x}^{2} d x d \tau \leq 0 .
$$

Next, we estimate the last term of the right-hand side of (3.2) by using (2.9) as follows:

$$
\begin{aligned}
\int_{0}^{t} \int_{\mathbb{R}} \tau^{\alpha} \phi_{\delta}(v) w_{x x} d x d \tau & \leq \int_{0}^{t} \tau^{\alpha}\left\|w_{x x}(\tau)\right\|_{L^{1}} d \tau \\
& \leq C \int_{0}^{t} \tau^{\alpha}(1+\tau)^{-1} d \tau \\
& \leq C(1+t)^{\alpha} .
\end{aligned}
$$

For the above claim, we can obtain the desired estimate (3.1).

The proof of Lemma 3.1 is complete.

Theorem 3.2 (Decay estimates). Suppose that $v_{0} \in H^{2} \cap L^{1}$. Then the Cauchy problem (2.14), (2.15) admits a unique global solution $v(t, x) \in X_{1}(0, \infty)$, and for any $0<\alpha<\frac{1}{7}, 0<\varepsilon<\frac{1}{2}$, the solution $v(t, x)$ satisfies

$$
\begin{aligned}
(1+t)^{\frac{1}{2}-\frac{3 \alpha}{2}+\varepsilon}\|v(t)\|^{2} & +\int_{0}^{t}(1+\tau)^{\frac{1}{2}-\frac{3 \alpha}{2}+\varepsilon}\left(\left\|\left(\sqrt{w_{x}} v\right)(\tau)\right\|^{2}+\tau^{\alpha}\left\|v_{x}(\tau)\right\|^{2}\right) d \tau \\
& \leq C(1+t)^{\varepsilon}\left(\left\|v_{0}\right\|_{L^{1}}^{2}+\left\|v_{0}\right\|^{2}+1\right)
\end{aligned}
$$

$$
\begin{aligned}
& (1+t)^{\frac{3}{2}-\alpha+\varepsilon}\left\|v_{x}(t)\right\|^{2}+\int_{0}^{t}(1+\tau)^{\frac{3}{2}-\alpha+\varepsilon}\left(\left\|\left(\sqrt{w_{x}} v_{x}\right)(\tau)\right\|^{2}+\tau^{\alpha}\left\|v_{x x}(\tau)\right\|^{2}\right) d \tau \\
& \leq C(1+t)^{\varepsilon}\left(\left\|v_{0}\right\|_{L^{1}}^{2}+\left\|v_{0}\right\|_{1}^{2}+1\right)^{5} \\
& (1+t)^{2-\alpha+\varepsilon}\left\|v_{x x}(t)\right\|^{2}+\int_{0}^{t}(1+\tau)^{2-\alpha+\varepsilon}\left(\left\|\left(\sqrt{w_{x}} v_{x x}\right)(\tau)\right\|^{2}+\tau^{\alpha}\left\|v_{x x x}(\tau)\right\|^{2}\right) d \tau \\
& \leq C(1+t)^{\varepsilon}\left(\left\|v_{0}\right\|_{L^{1}}^{2}+\left\|v_{0}\right\|_{2}^{2}+1\right)^{15} .
\end{aligned}
$$


Proof. First, if we have obtained the estimates (3.3), (3.4) and (3.5), then the global existence result on $v(t, x) \in X_{1}(0, \infty)$ will follow by the local existence result. Next, we will give the proofs of the estimates (3.3), (3.4) and (3.5).

Multiplying (2.14) by $v$ and integrating the resulting equation over $\mathbb{R}$, we have

$$
\begin{aligned}
\frac{1}{2} \frac{d}{d t}\|v(t)\|^{2}+\int_{\mathbb{R}}(f(v+w)-f(w))_{x} v d x & =\int_{\mathbb{R}} t^{\alpha} v_{x x} v d x+\int_{\mathbb{R}} t^{\alpha} w_{x x} v d x \\
& =I_{1}+I_{2} .
\end{aligned}
$$

By using (1.2), the second term of the left-hand side in (3.6) is estimated from below:

$$
\begin{aligned}
& \int_{\mathbb{R}}(f(v+w)-f(w))_{x} v d x \\
= & \int_{\mathbb{R}}\left\{-\left(\int_{w}^{v+w} f(s) d s-f(w) v\right)_{x}+\left(f(v+w)-f(w)-f^{\prime}(w) v\right) w_{x}\right\} d x \\
= & \int_{\mathbb{R}}\left(f(v+w)-f(w)-f^{\prime}(w) v\right) w_{x} d x \\
\geq & \frac{\beta}{2} \int_{\mathbb{R}} w_{x} v^{2} d x .
\end{aligned}
$$

Now we estimate $I_{1}$ as follows:

$$
I_{1}=-t^{\alpha} \int_{\mathbb{R}} v_{x}^{2} d x .
$$

For $I_{2}$, by the Sobolev inequality and Young's inequality, we get from (2.9),

$$
\begin{aligned}
I_{2} & \leq t^{\alpha}\|v(t)\|_{L^{\infty}}\left\|w_{x x}(t)\right\|_{L^{1}} \\
& \leq C t^{\alpha}(1+t)^{-1}\left\|v_{x}(t)\right\|\left\|^{\frac{1}{2}}\right\| v(t) \|^{\frac{1}{2}} \\
& \leq \frac{1}{2} t^{\alpha}\left\|v_{x}(t)\right\|^{2}+C t^{\alpha}(1+t)^{-\frac{4}{3}}\|v(t)\|^{\frac{2}{3}} .
\end{aligned}
$$

From (3.7)-(3.9), we can rewrite (3.6) as follows:

$$
\frac{1}{2} \frac{d}{d t}\|v(t)\|^{2}+\frac{\beta}{2}\left\|\left(\sqrt{w_{x}} v\right)(t)\right\|^{2}+\frac{1}{2} t^{\alpha}\left\|v_{x}(t)\right\|^{2} \leq C t^{\alpha}(1+t)^{-\frac{4}{3}}\|v(t)\|^{\frac{2}{3}} .
$$

Multiplying (3.10) by $(1+t)^{\frac{1}{2}-\frac{3 \alpha}{2}+\varepsilon}$, we have from Young's inequality,

$$
\begin{aligned}
\frac{1}{2} \frac{d}{d t} & \left((1+t)^{\frac{1}{2}-\frac{3 \alpha}{2}+\varepsilon}\|v(t)\|^{2}\right) \\
& +\frac{\beta}{2}(1+t)^{\frac{1}{2}-\frac{3 \alpha}{2}+\varepsilon}\left\|\left(\sqrt{w_{x}} v\right)(t)\right\|^{2} \\
& +\frac{1}{2} t^{\alpha}(1+t)^{\frac{1}{2}-\frac{3 \alpha}{2}+\varepsilon}\left\|v_{x}(t)\right\|^{2} \\
& \leq C(1+t)^{-\frac{1}{2}-\frac{3 \alpha}{2}+\varepsilon}\|v(t)\|^{2}+C t^{\alpha}(1+t)^{-\frac{5}{6}-\frac{3 \alpha}{2}+\varepsilon}\|v(t)\|^{\frac{2}{3}} \\
& \leq C t^{\frac{3 \alpha}{2}}(1+t)^{-1-\frac{3 \alpha}{2}+\varepsilon}+C(1+t)^{-\frac{1}{2}-\frac{3 \alpha}{2}+\varepsilon}\|v(t)\|^{2} .
\end{aligned}
$$


By using the Gagliardo-Nirenberg inequality, we have

$$
\|v(t)\|^{2} \leq C\|v(t)\|_{L^{1}}^{\frac{4}{3}}\left\|v_{x}(t)\right\|^{\frac{2}{3}} .
$$

Applying (3.1), (3.12) and Young's inequality to the last term of the right-hand side in (3.11), we have

$$
\begin{aligned}
C & (1+t)^{-\frac{1}{2}-\frac{3 \alpha}{2}+\varepsilon}\|v(t)\|^{2} \\
\leq & \frac{1}{4} t^{\alpha}(1+t)^{\frac{1}{2}-\frac{3 \alpha}{2}+\varepsilon}\left\|v_{x}(t)\right\|^{2} \\
& +C t^{-\frac{\alpha}{2}}(1+t)^{-1-\frac{3 \alpha}{2}+\varepsilon}\|v(t)\|_{L^{1}}^{2} \\
\leq & \frac{1}{4} t^{\alpha}(1+t)^{\frac{1}{2}-\frac{3 \alpha}{2}+\varepsilon}\left\|v_{x}(t)\right\|^{2} \\
& +C t^{-\frac{\alpha}{2}}(1+t)^{-1-\frac{3 \alpha}{2}+\varepsilon}\left\|v_{0}\right\|_{L^{1}}^{2} \\
& +C t^{-\frac{\alpha}{2}}(1+t)^{-1+\frac{\alpha}{2}+\varepsilon} .
\end{aligned}
$$

From (3.13), we integrate (3.11) over $[0, t]$ in $t$ to obtain (3.3).

Next, we derive the higher-order estimates. Multiplying $(2.14)$ by $\left(-v_{x x}\right)$ and integrating the resulting equation over $\mathbb{R}$, we have

$$
\frac{1}{2} \frac{d}{d t}\left\|v_{x}(t)\right\|^{2}-\int_{\mathbb{R}}(f(v+w)-f(w))_{x} v_{x x} d x=-t^{\alpha} \int_{\mathbb{R}} v_{x x}^{2} d x-t^{\alpha} \int_{\mathbb{R}} w_{x x} v_{x x} d x
$$

By using (1.2), the second term of (3.14) can be estimated as follows:

$$
\begin{aligned}
& -\int_{\mathbb{R}}(f(v+w)-f(w))_{x} v_{x x} d x \\
& \quad=-\int_{\mathbb{R}} f^{\prime}(v+w)\left(v_{x}+w_{x}\right) v_{x x} d x+\int_{\mathbb{R}} f^{\prime}(w) w_{x} v_{x x} d x \\
& \quad=\frac{1}{2} \int_{\mathbb{R}} f^{\prime \prime}(v+w)\left(v_{x}+w_{x}\right) v_{x}^{2} d x-\int_{\mathbb{R}} f^{\prime \prime}\left(\xi_{1}\right) v w_{x} v_{x x} d x \\
& \quad \geq \frac{\beta}{2} \int_{\mathbb{R}} w_{x} v_{x}^{2} d x+\frac{1}{2} \int_{\mathbb{R}} f^{\prime \prime}(v+w) v_{x}^{3} d x-\int_{\mathbb{R}} f^{\prime \prime}\left(\xi_{1}\right) v w_{x} v_{x x} d x,
\end{aligned}
$$

where $\xi_{1}$ is between $w$ and $v+w$.

Hence, (3.14) can be rewritten as

$$
\begin{aligned}
& \frac{1}{2} \frac{d}{d t}\left\|v_{x}(t)\right\|^{2}+\frac{\beta}{2} \int_{\mathbb{R}} w_{x} v_{x}^{2} d x+t^{\alpha} \int_{\mathbb{R}} v_{x x}^{2} d x \\
& \quad \leq C \int_{\mathbb{R}}\left|v_{x}^{3}\right| d x+C \int_{\mathbb{R}}\left|v w_{x} v_{x x}\right| d x+C t^{\alpha} \int_{\mathbb{R}}\left|w_{x x} v_{x x}\right| d x \\
& \quad=I_{3}+I_{4}+I_{5} .
\end{aligned}
$$

First, we estimate $I_{3}$ by using the Gagliardo-Nirenberg inequality and Young's inequality:

$$
I_{3} \leq\left. C\left\|v_{x x}(t)\right\|\right|^{\frac{7}{4}}\|v(t)\|^{\frac{5}{4}} \leq \frac{1}{4} t^{\alpha}\left\|v_{x x}(t)\right\|^{2}+C t^{-7 \alpha}\|v(t)\|^{10} .
$$


By using (3.3), we get

$$
\|v(t)\|^{2} \leq C(1+t)^{-\frac{1}{2}+\frac{3 \alpha}{2}}\left(\left\|v_{0}\right\|_{L^{1}}^{2}+\left\|v_{0}\right\|^{2}+1\right) .
$$

Thus

$$
I_{3} \leq \frac{1}{4} t^{\alpha}\left\|v_{x x}(t)\right\|^{2}+C t^{-7 \alpha}(1+t)^{-\frac{5}{2}+\frac{15 \alpha}{2}}\left(\left\|v_{0}\right\|_{L^{1}}^{2}+\left\|v_{0}\right\|^{2}+1\right)^{5} .
$$

The estimate of $I_{4}$ is as follows by (2.8) and (3.18):

$$
\begin{aligned}
I_{4} & \leq \frac{1}{4} t^{\alpha}\left\|v_{x x}(t)\right\|^{2}+C t^{-\alpha}\left\|w_{x}\right\|_{L^{\infty}}^{2}\|v(t)\|^{2} \\
& \leq \frac{1}{4} t^{\alpha}\left\|v_{x x}(t)\right\|^{2}+C t^{-\alpha}(1+t)^{-\frac{5}{2}+\frac{3 \alpha}{2}}\left(\left\|v_{0}\right\|_{L^{1}}^{2}+\left\|v_{0}\right\|^{2}+1\right) .
\end{aligned}
$$

For $I_{5}$, by using the Cauchy-Schwarz inequality, we have from (2.9),

$$
I_{5} \leq \frac{1}{4} t^{\alpha}\left\|v_{x x}(t)\right\|^{2}+C t^{\alpha}\left\|w_{x x}(t)\right\|^{2} \leq \frac{1}{4} t^{\alpha}\left\|v_{x x}(t)\right\|^{2}+C t^{\alpha}(1+t)^{-\frac{5}{2}} .
$$

Substituting (3.19)-(3.21) into (3.16), we have

$$
\begin{aligned}
\frac{1}{2} \frac{d}{d t}\left\|v_{x}(t)\right\|^{2}+ & \frac{\beta}{2}\left\|\left(\sqrt{w_{x}} v_{x}\right)(t)\right\|^{2}+\frac{1}{4} t^{\alpha}\left\|v_{x x}(t)\right\|^{2} \\
\leq & C t^{-7 \alpha}(1+t)^{-\frac{5}{2}+\frac{15 \alpha}{2}}\left(\left\|v_{0}\right\|_{L^{1}}^{2}+\left\|v_{0}\right\|^{2}+1\right)^{5} \\
& +C t^{-\alpha}(1+t)^{-\frac{5}{2}+\frac{3 \alpha}{2}}\left(\left\|v_{0}\right\|_{L^{1}}^{2}+\left\|v_{0}\right\|^{2}+1\right)+C t^{\alpha}(1+t)^{-\frac{5}{2}}
\end{aligned}
$$

Integrating $(3.22)$ over $[0, t]$ in $t$, we can obtain

$$
\left\|v_{x}(t)\right\|^{2}+\int_{0}^{t}\left(\left\|\left(\sqrt{w_{x}} v_{x}\right)(\tau)\right\|^{2}+\tau^{\alpha}\left\|v_{x x}(\tau)\right\|^{2}\right) d \tau \leq C\left(\left\|v_{0}\right\|_{L^{1}}^{2}+\left\|v_{0}\right\|^{2}+1\right)^{5} .
$$

By (3.3) and (3.23), we have for $t>1$,

$$
\begin{aligned}
\int_{0}^{t}(1 & +\tau)^{\frac{1}{2}-\alpha+\varepsilon}\left\|v_{x}(\tau)\right\|^{2} d \tau \\
& \leq \int_{0}^{1}(1+\tau)^{\frac{1}{2}-\alpha+\varepsilon}\left\|v_{x}(\tau)\right\|^{2} d \tau+C \int_{1}^{t} \tau^{\alpha}(1+\tau)^{\frac{1}{2}-\frac{3}{2} \alpha+\varepsilon}\left\|v_{x}(\tau)\right\|^{2} d \tau \\
& \leq C(1+t)^{\varepsilon}\left(\left\|v_{0}\right\|_{L^{1}}^{2}+\left\|v_{0}\right\|^{2}+1\right) .
\end{aligned}
$$


Multiplying (3.22) by $(1+t)^{\frac{3}{2}-\alpha+\varepsilon}$ and integrating the resulting inequality over $[0, t]$ in $t$, we have from the above inequality:

$$
\begin{aligned}
& (1+t)^{\frac{3}{2}-\alpha+\varepsilon}\left\|v_{x}(t)\right\|^{2}+\int_{0}^{t}(1+\tau)^{\frac{3}{2}-\alpha+\varepsilon}\left(\left\|\left(\sqrt{w_{x}} v_{x}\right)(\tau)\right\|^{2}+\tau^{\alpha}\left\|v_{x x}(\tau)\right\|^{2}\right) d \tau \\
\leq & C\left\|v_{0 x}\right\|^{2}+C \int_{0}^{t}(1+\tau)^{\frac{1}{2}-\alpha+\varepsilon}\left\|v_{x}(\tau)\right\|^{2} d \tau \\
& +C\left(\left\|v_{0}\right\|_{L^{1}}^{2}+\left\|v_{0}\right\|^{2}+1\right)^{5} \int_{0}^{t} \tau^{-7 \alpha}(1+\tau)^{-1+\frac{13 \alpha}{2}+\varepsilon} d \tau \\
& +C\left(\left\|v_{0}\right\|_{L^{1}}^{2}+\left\|v_{0}\right\|^{2}+1\right) \int_{0}^{t} \tau^{-\alpha}(1+\tau)^{-1+\frac{\alpha}{2}+\varepsilon} d \tau \\
& +C \int_{0}^{t} \tau^{\alpha}(1+\tau)^{-1-\alpha+\varepsilon} d \tau \\
\leq & C(1+t)^{\varepsilon}\left(\left\|v_{0}\right\|_{L^{1}}^{2}+\left\|v_{0}\right\|_{1}^{2}+1\right)^{5}
\end{aligned}
$$

which implies (3.4).

Differentiating (2.14) twice in $x$ and multiplying it by $v_{x x}$, we have

$$
\begin{gathered}
\frac{1}{2} \frac{d}{d t}\left\|v_{x x}(t)\right\|^{2}+\int_{\mathbb{R}} v_{x x}(f(v+w)-f(w))_{x x x} d x \\
=t^{\alpha} \int_{\mathbb{R}} v_{x x x x} v_{x x} d x+t^{\alpha} \int_{\mathbb{R}} w_{x x x x} v_{x x} d x .
\end{gathered}
$$

We first study the second term in (3.24). Integration by parts gives $(3.25)$

$$
\begin{aligned}
& \int_{\mathbb{R}} v_{x x}(f(v+w)-f(w))_{x x x} d x=-\int_{\mathbb{R}} v_{x x x}(f(v+w)-f(w))_{x x} d x \\
& =\frac{1}{2} \int_{\mathbb{R}} f^{\prime \prime}(v+w) v_{x} v_{x x}^{2} d x+\frac{1}{2} \int_{\mathbb{R}} f^{\prime \prime}(v+w) w_{x} v_{x x}^{2} d x-\int_{\mathbb{R}} f^{\prime \prime \prime}\left(\xi_{2}\right) w_{x}^{2} v v_{x x x} d x \\
& -2 \int_{\mathbb{R}} f^{\prime \prime}(v+w) v_{x} w_{x} v_{x x x} d x-\int_{\mathbb{R}} f^{\prime \prime}(v+w) v_{x}^{2} v_{x x x} d x-\int_{\mathbb{R}} f^{\prime \prime}\left(\xi_{1}\right) v w_{x x} v_{x x x} d x
\end{aligned}
$$

where $\xi_{1}$ is defined by (3.15) and $\xi_{2}$ is between $w$ and $v+w$.

Hence, (3.24) can be rewritten as

$$
\begin{aligned}
\frac{1}{2} \frac{d}{d t}\left\|v_{x x}(t)\right\|^{2}+\frac{\beta}{2} \int_{\mathbb{R}} w_{x} v_{x x}^{2} d x+t^{\alpha} \int_{\mathbb{R}} v_{x x x}^{2} d x \\
\leq C t^{\alpha} \int_{\mathbb{R}}\left|w_{x x x} v_{x x x}\right| d x+C \int_{\mathbb{R}}\left|v_{x x}^{2} v_{x}\right| d x+C \int_{\mathbb{R}}\left|v w_{x}^{2} v_{x x x}\right| d x \\
\quad+C \int_{\mathbb{R}}\left|w_{x x} v v_{x x x}\right| d x+C \int_{\mathbb{R}}\left|v_{x} w_{x} v_{x x x}\right| d x+C \int_{\mathbb{R}}\left|v_{x}^{2} v_{x x x}\right| d x \\
=I_{6}+I_{7}+I_{8}+I_{9}+I_{10}+I_{11} .
\end{aligned}
$$


Next, we will give the estimates of $I_{6}-I_{11}$, respectively. For $I_{6}$, by using the Cauchy-Schwarz inequality and (2.10), we get

$$
\begin{aligned}
I_{6} & \leq \frac{1}{8} t^{\alpha}\left\|v_{x x x}(t)\right\|^{2}+C t^{\alpha}\left\|w_{x x x}(t)\right\|^{2} \\
& \leq \frac{1}{8} t^{\alpha}\left\|v_{x x x}(t)\right\|^{2}+C t^{\alpha}(1+t)^{-3} .
\end{aligned}
$$

We compute $I_{7}$ as follows by the Hölder inequality:

$$
I_{7} \leq C|| v_{x}(t)|||| v_{x x}(t) \|_{L^{4}}^{2} .
$$

By the Gagliardo-Nirenberg inequality, we have

$$
\left\|v_{x x}(t)\right\|_{L^{4}}^{2} \leq C|| v_{x x x}(t)\|\| v_{x}(t) \|_{\infty} .
$$

Therefore, the Sobolev inequality, Young's inequality and (3.4) give (3.28)

$$
\begin{aligned}
I_{7} \leq & C\left\|v_{x}(t)\right\|\left\|v_{x x x}(t)\right\|\left\|v_{x}(t)\right\|_{L^{\infty}} \\
\leq & C\left\|v_{x}(t)\right\|\left\|^{\frac{3}{2}}\right\| v_{x x x}(t)\|\| v_{x x}(t) \|^{\frac{1}{2}} \\
\leq & \frac{1}{8} t^{\alpha}\left\|v_{x x x}(t)\right\|^{2}+C t^{\alpha}(1+t)^{-\frac{1}{2}}\left\|v_{x x}(t)\right\|^{2}+C t^{-3 \alpha}(1+t)^{\frac{1}{2}}\left\|v_{x}(t)\right\|^{6} \\
\leq & \frac{1}{8} t^{\alpha}\left\|v_{x x x}(t)\right\|^{2}+C t^{\alpha}(1+t)^{-\frac{1}{2}}\left\|v_{x x}(t)\right\|^{2} \\
& +C t^{-3 \alpha}(1+t)^{-4+3 \alpha}\left(\left\|v_{0}\right\|_{L^{1}}^{2}+\left\|v_{0}\right\|_{1}^{2}+1\right)^{15} .
\end{aligned}
$$

For $I_{8}$, by using the Cauchy-Schwarz inequality, we get from (3.3) and (2.8),

$$
\begin{aligned}
I_{8} & \leq \frac{1}{8} t^{\alpha}\left\|v_{x x x}(t)\right\|^{2}+C t^{-\alpha}\left\|w_{x}(t)\right\|_{L^{\infty}}^{4}\|v(t)\|^{2} \\
& \leq \frac{1}{8} t^{\alpha}\left\|v_{x x x}(t)\right\|^{2}+C t^{-\alpha}(1+t)^{-\frac{9}{2}+\frac{3 \alpha}{2}}\left(\left\|v_{0}\right\|_{L^{1}}^{2}+\left\|v_{0}\right\|_{1}^{2}+1\right) .
\end{aligned}
$$

Similarly, we have from (2.9) and (3.3), (3.4),

$$
\begin{aligned}
I_{9} & \leq \frac{1}{8} t^{\alpha}\left\|v_{x x x}(t)\right\|^{2}+C t^{-\alpha}\left\|w_{x x}(t)\right\|_{L^{\infty}}^{2}\|v(t)\|^{2} \\
& \leq \frac{1}{8} t^{\alpha}\left\|v_{x x x}(t)\right\|^{2}+C t^{-\alpha}(1+t)^{-\frac{7}{2}+\frac{3 \alpha}{2}}\left(\left\|v_{0}\right\|_{L^{1}}^{2}+\left\|v_{0}\right\|_{1}^{2}+1\right)
\end{aligned}
$$

and

$$
\begin{aligned}
I_{10} & \leq \frac{1}{8} t^{\alpha}\left\|v_{x x x}(t)\right\|^{2}+C t^{-\alpha}\left\|w_{x}(t)\right\|_{L^{\infty}}^{2}\left\|v_{x}(t)\right\|^{2} \\
& \leq \frac{1}{8} t^{\alpha}\left\|v_{x x x}(t)\right\|^{2}+C t^{-\alpha}(1+t)^{-\frac{7}{2}+\alpha}\left(\left\|v_{0}\right\|_{L^{1}}^{2}+\left\|v_{0}\right\|_{1}^{2}+1\right)^{5} .
\end{aligned}
$$


Similar to the estimate of $I_{7}$, we estimate $I_{11}$ as follows:

$$
\begin{aligned}
I_{11} \leq & C\left\|v_{x x x}(t)\right\|\left\|v_{x}(t)\right\|_{L^{4}}^{2} \\
\leq & C\left\|v_{x x x}(t)\right\|\left\|v_{x}(t)\right\|_{L^{\infty}}\left\|v_{x}(t)\right\| \\
\leq & \frac{1}{8} t^{\alpha}\left\|v_{x x x}(t)\right\|^{2}+C t^{\alpha}(1+t)^{-\frac{1}{2}}\left\|v_{x x}(t)\right\|^{2} \\
& +C t^{-3 \alpha}(1+t)^{-4+3 \alpha}\left(\left\|v_{0}\right\|_{L^{1}}^{2}+\left\|v_{0}\right\|_{1}^{2}+1\right)^{15} .
\end{aligned}
$$

Substituting (3.27)-(3.32) into (3.26), we have

$$
\begin{aligned}
& \frac{1}{2} \frac{d}{d t}\left\|v_{x x}(t)\right\|^{2}+\frac{\beta}{2} \int_{\mathbb{R}} w_{x} v_{x x}^{2} d x+t^{\alpha} \int_{\mathbb{R}} v_{x x x}^{2} d x \\
& \leq C t^{\alpha}(1+t)^{-3}+C t^{-\alpha}(1+t)^{-\frac{7}{2}+\frac{3 \alpha}{2}}\left(\left\|v_{0}\right\|_{L^{1}}^{2}+\left\|v_{0}\right\|_{1}^{2}+1\right)^{5} \\
& \quad+C t^{\alpha}(1+t)^{-\frac{1}{2}}\left\|v_{x x}(t)\right\|^{2}+C t^{-3 \alpha}(1+t)^{-4+3 \alpha}\left(\left\|v_{0}\right\|_{L^{1}}^{2}+\left\|v_{0}\right\|_{1}^{2}+1\right)^{15} .
\end{aligned}
$$

Integrating (3.33) over $[0, t]$ in $t$, we have from (3.23),

$$
\left\|v_{x x}(t)\right\|^{2}+\int_{0}^{t}\left(\left\|\left(\sqrt{w_{x}} v_{x x}\right)(\tau)\right\|^{2}+\tau^{\alpha}\left\|v_{x x x}(\tau)\right\|^{2}\right) d \tau \leq C\left(\left\|v_{0}\right\|_{L^{1}}^{2}+\left\|v_{0}\right\|_{2}^{2}+1\right)^{15}
$$

Multiplying (3.33) by $(1+t)^{2-\alpha+\varepsilon}$, and integrating the resulting inequality over $[0, t]$ in $t$, we can obtain (3.5) by (3.4).

The proof of Theorem 3.2 is complete.

\section{Decay estimates for the perturbation $V(t, x, y)$}

For any $0<T \leq \infty$, define

$$
X_{2}(0, T)=\left\{V(t, x, y): V \in L^{\infty}\left([0, T] ; H^{2}\right), t^{\frac{\alpha}{2}} \nabla V \in L^{2}\left([0, T] ; H^{2}\right)\right\} .
$$

Suppose that $V_{0} \in H^{2}$. By a standard argument, we can obtain $V(t, x, y) \in$ $X_{2}\left(0, t_{1}\right)$, where $t_{1}$ depends on $\left\|V_{0}\right\|_{2}$.

Therefore, in order to get the global existence of solutions to (2.16) and (2.17) in $X_{2}(0, \infty)$, it is sufficient to get a priori estimates in $X_{2}(0, \infty)$. To do this, we need to establish the $L^{1}$-estimate on $V(t, x, y)$.

Lemma 4.1 ( $L^{1}$-estimate). Suppose further that $V_{0} \in L^{1} \cap H^{2}$. Then the solution $V(t, x, y)$ of (2.16) and (2.17) satisfies

$$
\|V(t)\|_{L^{1}} \leq\left\|V_{0}\right\|_{L^{1}}
$$

The proof is similar to that in Lemma 3.1, and the details are omitted.

Theorem 4.2 (Decay estimate). Let $V_{0} \in H^{2} \cap L^{1}$. Then the Cauchy problem (2.16) and (2.17) admits a unique global solution $V(t, x, y) \in X_{2}(0, \infty)$, and for any $0<\alpha<\frac{1}{7}, 0<\varepsilon<\frac{1}{2}$, the solution $V(t, x, y)$ satisfies:

$$
\begin{gathered}
(1+t)^{1+\alpha+\varepsilon}\|V(t)\|^{2}+\int_{0}^{t}(1+\tau)^{1+\alpha+\varepsilon}\left(\left\|\left(\sqrt{U_{x}} V\right)(\tau)\right\|^{2}+\tau^{\alpha}\|\nabla V(\tau)\|^{2}\right) d \tau \\
\leq C(1+t)^{\varepsilon}\left(\left\|V_{0}\right\|_{L^{1}}^{2}+\left\|V_{0}\right\|^{2}+1\right)
\end{gathered}
$$




$$
\begin{gathered}
(1+t)^{2+\alpha+\varepsilon}\left\|V_{y}(t)\right\|^{2}+\int_{0}^{t}(1+\tau)^{2+\alpha+\varepsilon}\left(\left\|\left(\sqrt{U_{x}} V_{y}\right)(\tau)\right\|^{2}+\tau^{\alpha}\left\|\nabla V_{y}(\tau)\right\|^{2}\right) d \tau \\
\leq C(1+t)^{\varepsilon}\left(\left\|V_{0}\right\|_{L^{1}}^{2}+\left\|V_{0}\right\|_{1}^{2}+1\right)^{2}
\end{gathered}
$$

$$
\begin{gathered}
(1+t)^{\frac{15}{8}+\frac{\alpha}{2}+\varepsilon}\left\|V_{x}(t)\right\|^{2}+\int_{0}^{t}(1+\tau)^{\frac{15}{8}+\frac{\alpha}{2}+\varepsilon}\left(\left\|\left(\sqrt{U_{x}} V_{x}\right)(\tau)\right\|^{2}+\tau^{\alpha}\left\|\nabla V_{x}(\tau)\right\|^{2}\right) d \tau \\
\leq C(1+t)^{\varepsilon}\left(\left\|V_{0}\right\|_{L^{1}}^{2}+\left\|V_{0}\right\|_{1}^{2}+1\right)^{2},
\end{gathered}
$$

$(1+t)^{3+\alpha+\varepsilon}\left\|V_{y y}(t)\right\|^{2}+\int_{0}^{t}(1+\tau)^{3+\alpha+\varepsilon}\left(\left\|\left(\sqrt{U_{x}} V_{y y}\right)(\tau)\right\|^{2}+\tau^{\alpha}\left\|\nabla V_{y y}(\tau)\right\|^{2}\right) d \tau$

$$
\leq C(1+t)^{\varepsilon}\left(\left\|V_{0}\right\|_{L^{1}}^{2}+\left\|V_{0}\right\|_{2}^{2}+1\right)^{10}
$$

$$
\begin{gathered}
(1+t)^{\frac{23}{8}+\frac{\alpha}{2}+\varepsilon}\left\|V_{x y}(t)\right\|^{2}+\int_{0}^{t}(1+\tau)^{\frac{23}{8}+\frac{\alpha}{2}+\varepsilon}\left(\left\|\left(\sqrt{U_{x}} V_{x y}\right)(\tau)\right\|^{2}\right. \\
\left.+\tau^{\alpha}\left\|\nabla V_{x y}(\tau)\right\|^{2}\right) d \tau \\
\leq C(1+t)^{\varepsilon}\left(\left\|V_{0}\right\|_{L^{1}}^{2}+\left\|V_{0}\right\|_{2}^{2}+1\right)^{14}, \\
(1+t)^{\frac{39}{16}+\frac{3 \alpha}{2}+\varepsilon}\left\|V_{x x}(t)\right\|^{2}+\int_{0}^{t}(1+\tau)^{\frac{39}{16}+\frac{3 \alpha}{2}+\varepsilon}\left(\left\|\left(\sqrt{U_{x}} V_{x x}\right)(\tau)\right\|^{2}\right. \\
\left.+\tau^{\alpha}\left\|\nabla V_{x x}(\tau)\right\|^{2}\right) d \tau \\
\leq C(1+t)^{\varepsilon}\left(\left\|V_{0}\right\|_{L^{1}}^{2}+\left\|V_{0}\right\|_{2}^{2}+1\right)^{10} .
\end{gathered}
$$

Corollary 4.3. From (4.1) and (4.2), we obtain

$$
\|V(t)\|^{2} \leq C(1+t)^{-1-\alpha}, \quad\left\|V_{y}(t)\right\|^{2} \leq C(1+t)^{-2-\alpha},
$$

which implies by the Sobolev inequality that

$$
\sup _{y \in \mathbb{R}}\|V(t, \cdot, y)\|_{L^{2}\left(\mathbb{R}_{x}\right)} \leq C(1+t)^{-\frac{3}{4}-\frac{\alpha}{2}} .
$$

Next, we will give the proof of Theorem 4.2.

Proof of Theorem 4.2. Multiplying (2.16) by $V$ and integrating the resulting equation over $\mathbb{R}^{2}$, we have

$$
\begin{aligned}
\frac{1}{2} \frac{d}{d t}\|V(t)\|^{2} & +\int_{\mathbb{R}^{2}}(f(V+U)-f(U))_{x} V d x d y+\int_{\mathbb{R}^{2}} g(U+V)_{y} V d x d y \\
& =\int_{\mathbb{R}^{2}} t^{\alpha} V_{x x} V d x d y+\int_{\mathbb{R}^{2}} t^{\alpha} V_{y y} V d x d y .
\end{aligned}
$$

Similar to (3.7), we have

$$
\int_{\mathbb{R}^{2}}(f(U+V)-f(U))_{x} V d x d y \geq \frac{\beta}{2} \int_{\mathbb{R}^{2}} U_{x} V^{2} d x d y .
$$


On the other hand,

$$
\begin{aligned}
\int_{\mathbb{R}^{2}} g(U+V)_{y} V d x d y & =-\int_{\mathbb{R}^{2}} g(U+V) V_{y} d x d y \\
& =-\int_{\mathbb{R}^{2}}\left(g(U+V)(U+V)_{y}-g(U) U_{y}\right) d x d y \\
& =-\int_{\mathbb{R}^{2}}\left(\frac{\partial}{\partial y} \int_{U}^{U+V} g(\xi) d \xi\right) d x d y \\
& =0 .
\end{aligned}
$$

From (4.8)-(4.10), we have

$$
\frac{1}{2} \frac{d}{d t}\|V(t)\|^{2}+\frac{\beta}{2}\left\|\left(\sqrt{U_{x}} V\right)(t)\right\|^{2}+t^{\alpha}\|\nabla V(t)\|^{2} \leq 0 .
$$

Multiplying (4.11) by $(1+t)^{1+\alpha+\varepsilon}$, we have

$$
\begin{array}{cc}
\frac{1}{2} \frac{d}{d t}\left((1+t)^{1+\alpha+\varepsilon}\|V(t)\|^{2}\right)+\frac{\beta}{2}(1+t)^{1+\alpha+\varepsilon} \mid\left\|\left(\sqrt{U_{x}} V\right)(t)\right\|^{2} \\
+(1+t)^{1+\alpha+\varepsilon} t^{\alpha}\|\nabla V(t)\|^{2} \\
\leq C(1+t)^{\alpha+\varepsilon}\|V(t)\|^{2} .
\end{array}
$$

By the Gagliardo-Nirenberg inequality

$$
\|V(t)\|^{2} \leq C\|V(t)\|_{L^{1}}\|\nabla V(t)\|,
$$

we obtain from Lemma 4.1,

$$
\begin{gathered}
\frac{1}{2} \frac{d}{d t}\left((1+t)^{1+\alpha+\varepsilon}\|V(t)\|^{2}\right)+\frac{\beta}{2}(1+t)^{1+\alpha+\varepsilon}\left\|\left(\sqrt{U_{x}} V\right)(t)\right\|^{2} \\
+(1+t)^{1+\alpha+\varepsilon} t^{\alpha}\|\nabla V(t)\|^{2} \\
\leq \frac{1}{2} t^{\alpha}(1+t)^{1+\alpha+\varepsilon}\|\nabla V(t)\|^{2}+C t^{-\alpha}(1+t)^{\alpha-1+\varepsilon}\left\|V_{0}\right\|_{L^{1}}^{2} .
\end{gathered}
$$

Integrating (4.13) over $[0, t]$ in $t$, we obtain (4.1).

Before obtaining the decay rates of $V_{y}$ and $V_{x}$, we first establish the $H^{1}$-estimate of $V$. Multiplying (2.16) by $-V_{y y}$, and integrating the resulting equation over $\mathbb{R}^{2}$, we have

$$
\begin{aligned}
\frac{1}{2} \frac{d}{d t}\left\|V_{y}(t)\right\|^{2} & -\int_{\mathbb{R}^{2}}(f(V+U)-f(U))_{x} V_{y y} d x d y-\int_{\mathbb{R}^{2}} g(U+V)_{y} V_{y y} d x d y \\
& =-\int_{\mathbb{R}^{2}} t^{\alpha} V_{x x} V_{y y} d x d y-\int_{\mathbb{R}^{2}} t^{\alpha} V_{y y}^{2} d x d y .
\end{aligned}
$$

We estimate the second term of (4.14) as follows:

$$
\begin{aligned}
\int_{\mathbb{R}^{2}} & (f(V+U)-f(U))_{x} V_{y y} d x d y \\
& =\int_{\mathbb{R}^{2}}\left(f^{\prime}(V+U) V_{x}+f^{\prime \prime}\left(\xi_{3}\right) V U_{x}\right) V_{y y} d x d y \\
& \leq \frac{1}{4} t^{\alpha}\left\|V_{y y}(t)\right\|^{2}+C t^{-\alpha}\left\|V_{x}(t)\right\|^{2}+C t^{-\alpha}\left\|\left(U_{x} V\right)(t)\right\|^{2},
\end{aligned}
$$


where $\xi_{3}$ is between $U$ and $U+V$. The Gagliardo-Nirenberg inequality

$$
\begin{aligned}
\left\|V_{x}(t)\right\|^{2} & =\int_{\mathbb{R}}\left\|V_{x}(t)\right\|_{L^{2}\left(\mathbb{R}_{x}\right)}^{2} d y \\
& \leq \int_{\mathbb{R}}\left\|V_{x x}(t)\right\|_{L^{2}\left(\mathbb{R}_{x}\right)}\|V(t)\|_{L^{2}\left(\mathbb{R}_{x}\right)} d y \\
& \leq\left\|V_{x x}(t)\right\|\|V(t)\|
\end{aligned}
$$

gives us that

$$
\begin{aligned}
C t^{-\alpha}\left\|V_{x}(t)\right\|^{2} & \leq C t^{-\alpha}\left\|V_{x x}(t)|\||| V(t)\|\right. \\
& \leq \frac{1}{8} t^{\alpha}\left\|V_{x x}(t)\right\|^{2}+C t^{-3 \alpha}\|V(t)\|^{2} \\
& \leq \frac{1}{8} t^{\alpha}\left\|V_{x x}(t)\right\|^{2}+C t^{-3 \alpha}(1+t)^{-1-\alpha}\left(\left\|V_{0}\right\|_{L^{1}}^{2}+\left\|V_{0}\right\|^{2}\right) .
\end{aligned}
$$

Recalling $U=w+v$, and noticing (3.4), (3.5), (2.8), we have by the Sobolev inequality,

$$
\begin{aligned}
\left\|U_{x}(t)\right\|_{L^{\infty}} & \leq\left\|w_{x}(t)\right\|_{L^{\infty}}+\left\|v_{x}(t)\right\|_{L^{\infty}} \\
& \leq\left\|w_{x}(t)\right\|_{L^{\infty}}+\left\|v_{x x}(t)\right\|^{\frac{1}{2}}\left\|v_{x}(t)\right\|^{\frac{1}{2}} \\
& \leq C(1+t)^{-\frac{7}{8}+\frac{\alpha}{2}} .
\end{aligned}
$$

Therefore,

$$
\begin{aligned}
C t^{-\alpha}\left\|\left(U_{x} V\right)(t)\right\|^{2} & \leq C t^{-\alpha}\left\|U_{x}(t)\right\|_{L^{\infty}}^{2}\|V(t)\|^{2} \\
& \leq C t^{-\alpha}(1+t)^{-\frac{11}{4}}\left(\left\|V_{0}\right\|_{L^{1}}^{2}+\left\|V_{0}\right\|^{2}+1\right) .
\end{aligned}
$$

From (4.15)-(4.18), we have

$$
\begin{aligned}
\int_{\mathbb{R}^{2}}(f(V+ & U)-f(U))_{x} V_{y y} d x d y \\
\leq & \frac{1}{4} t^{\alpha}\left\|V_{y y}(t)\right\|^{2}+\frac{1}{8} t^{\alpha}\left\|V_{x x}(t)\right\|^{2} \\
& +C\left(t^{-3 \alpha}(1+t)^{-1-\alpha}+t^{-\alpha}(1+t)^{-\frac{11}{4}}\right)\left(\left\|V_{0}\right\|_{L^{1}}^{2}+\left\|V_{0}\right\|^{2}+1\right) .
\end{aligned}
$$

Similarly, the third term of (4.14) can be estimated as

$$
\begin{array}{rl}
\int_{\mathbb{R}^{2}} & g(U+V)_{y} V_{y y} d x d y \\
& \leq \frac{1}{8} t^{\alpha}\left\|V_{y y}(t)\right\|^{2}+C t^{-\alpha}\left\|V_{y}(t)\right\|^{2} \\
& \leq \frac{1}{8} t^{\alpha}\left\|V_{y y}(t)\right\|^{2}+C t^{-\alpha}\left\|V_{y y}(t)|\|\mid V(t)\|\right. \\
& \leq \frac{1}{4} t^{\alpha}\left\|V_{y y}(t)\right\|^{2}+C t^{-3 \alpha}(1+t)^{-1-\alpha}\left(\left\|V_{0}\right\|_{L^{1}}^{2}+\left\|V_{0}\right\|^{2}+1\right) .
\end{array}
$$


Substituting (4.19) and (4.20) into (4.14), we have (4.21)

$$
\begin{aligned}
& \frac{1}{2} \frac{d}{d t}\left\|V_{y}(t)\right\|^{2}+\frac{1}{2} t^{\alpha}\left\|\nabla V_{y}(t)\right\|^{2} \\
& \quad \leq \frac{1}{8} t^{\alpha}\left\|V_{x x}(t)\right\|^{2}+C\left(t^{-3 \alpha}(1+t)^{-1-\alpha}+t^{-\alpha}(1+t)^{-\frac{11}{4}}\right)\left(\left\|V_{0}\right\|_{L^{1}}^{2}+\left\|V_{0}\right\|^{2}+1\right) .
\end{aligned}
$$

Similar to (4.21), we have $(4.22)$

$$
\begin{aligned}
& \frac{1}{2} \frac{d}{d t}\left\|V_{x}(t)\right\|^{2}+\frac{1}{2} t^{\alpha}\left\|\nabla V_{x}(t)\right\|^{2} \\
& \quad \leq \frac{1}{8} t^{\alpha}\left\|V_{y y}(t)\right\|^{2}+C\left(t^{-3 \alpha}(1+t)^{-1-\alpha}+t^{-\alpha}(1+t)^{-\frac{11}{4}}\right)\left(\left\|V_{0}\right\|_{L^{1}}^{2}+\left\|V_{0}\right\|^{2}+1\right) .
\end{aligned}
$$

Adding (4.21) to (4.22) and integrating the resulting inequality over $[0, t]$ in $t$, we can obtain

$$
\begin{aligned}
\left\|V_{y}(t)\right\|^{2} & +\left\|V_{x}(t)\right\|^{2}+\int_{0}^{t} \tau^{\alpha}\left(\left\|\nabla V_{y}(\tau)\right\|^{2}+\left\|\nabla V_{x}(\tau)\right\|^{2}\right) d \tau \\
& \leq C\left(\left\|V_{0}\right\|_{L^{1}}^{2}+\left\|V_{0}\right\|_{1}^{2}+1\right) .
\end{aligned}
$$

Next, we will show the decay rate of $V_{y}$. An integration by parts gives that

the second term of (4.14)

$$
\begin{aligned}
& =\int_{\mathbb{R}^{2}}(f(U+V)-f(U))_{x y} V_{y} d x d y \\
& =\frac{1}{2} \int_{\mathbb{R}^{2}} f^{\prime \prime}(U+V) V_{y}^{2} U_{x} d x d y+\frac{1}{2} \int_{\mathbb{R}^{2}} f^{\prime \prime}(U+V) V_{y}^{2} V_{x} d x d y \\
& \geq \frac{\beta}{2} \int_{\mathbb{R}^{2}} U_{x} V_{y}^{2} d x d y+\frac{1}{2} \int_{\mathbb{R}^{2}} f^{\prime \prime}(U+V) V_{x} V_{y}^{2} d x d y,
\end{aligned}
$$

and

$$
\text { the third term of (4.14) }
$$

$$
\begin{aligned}
& =\int_{\mathbb{R}^{2}} g(U+V)_{y y} V_{y} d x d y \\
& =\int_{\mathbb{R}^{2}} g^{\prime \prime}(U+V) V_{y}^{3} d x d y+\int_{\mathbb{R}^{2}} g^{\prime}(U+V) V_{y y} V_{y} d x d y \\
& =\frac{1}{2} \int_{\mathbb{R}^{2}} g^{\prime \prime}(U+V) V_{y}^{3} d x d y .
\end{aligned}
$$

From (4.24), (4.25), we rewrite (4.14) as

$$
\begin{aligned}
\frac{1}{2} \frac{d}{d t} & \left\|V_{y}(t)\right\|^{2}+\frac{\beta}{2}\left\|\left(\sqrt{U}_{x} V_{y}\right)(t)\right\|^{2}+t^{\alpha}\left\|\nabla V_{y}(t)\right\|^{2} \\
& \leq C \int_{\mathbb{R}^{2}}\left|V_{x} V_{y}^{2}\right| d x d y+C \int_{\mathbb{R}^{2}}\left|V_{y}^{3}\right| d x d y \\
& =I_{12}+I_{13} .
\end{aligned}
$$


We estimate $I_{12}$ by using the Sobolev inequality and Young's inequality as follows:

$$
\begin{aligned}
I_{12} & \leq C \int_{\mathbb{R}}\left\|V_{x}(t)\right\|_{L^{\infty}\left(\mathbb{R}_{y}\right)}\left\|V_{y}(t)\right\|_{L^{2}\left(\mathbb{R}_{y}\right)}^{2} d x \\
& \leq C \int_{\mathbb{R}}\left\|V_{x y}(t)\right\|_{L^{2}\left(\mathbb{R}_{y}\right)}^{\frac{1}{2}}\left\|V_{x}(t)\right\|_{L^{2}\left(\mathbb{R}_{y}\right)}^{\frac{1}{2}}\left\|V_{y}(t)\right\|_{L^{2}\left(\mathbb{R}_{y}\right)}^{2} d x \\
& \leq \frac{1}{2} t^{\alpha}\left\|V_{x y}(t)\right\|^{2}+C t^{-\frac{\alpha}{3}} \int_{\mathbb{R}}\left\|V_{x}(t)\right\|_{L^{2}\left(\mathbb{R}_{y}\right)}^{\frac{2}{3}}\left\|V_{y}(t)\right\|_{L^{2}\left(\mathbb{R}_{y}\right)}^{\frac{8}{3}} d x .
\end{aligned}
$$

By the Gagliardo-Nirenberg inequality

$$
\left\|V_{y}(t)\right\|_{L^{2}\left(\mathbb{R}_{y}\right)}^{\frac{8}{3}} \leq\left\|V_{y y}(t)\right\|_{L^{2}\left(\mathbb{R}_{y}\right)}^{\frac{4}{3}}\|V(t)\|_{L^{2}\left(\mathbb{R}_{y}\right)}^{\frac{4}{3}},
$$

we obtain

$$
\begin{aligned}
I_{12} \leq \frac{1}{2} t^{\alpha}\left\|V_{x y}(t)\right\|^{2} & +C t^{-\frac{\alpha}{3}} \int_{\mathbb{R}}\left\|V_{x}(t)\right\|_{L^{2}\left(\mathbb{R}_{y}\right)}^{\frac{2}{3}}\left\|V_{y y}(t)\right\|_{L^{2}\left(\mathbb{R}_{y}\right)}^{\frac{4}{3}}\|V(t)\|_{L^{2}\left(\mathbb{R}_{y}\right)}^{\frac{4}{3}} d x \\
\leq \frac{1}{2} t^{\alpha}\left\|V_{x y}(t)\right\|^{2} & +\frac{1}{4} t^{\alpha}\left\|V_{y y}(t)\right\|^{2} \\
& +C t^{-3 \alpha} \int_{\mathbb{R}}\left\|V_{x}(t)\right\|_{L^{2}\left(\mathbb{R}_{y}\right)}^{2}\|V(t)\|_{L^{2}\left(\mathbb{R}_{y}\right)}^{4} d x \\
\leq \frac{1}{2} t^{\alpha}\left\|V_{x y}(t)\right\|^{2} & +\frac{1}{4} t^{\alpha}\left\|V_{y y}(t)\right\|^{2} \\
& +C t^{-3 \alpha}\left(\sup _{x \in \mathbb{R}}\|V(t)\|_{L^{2}\left(\mathbb{R}_{y}\right)}^{2}\right)^{2}\left\|V_{x}(t)\right\|^{2} \\
\leq \frac{1}{2} t^{\alpha}\left\|V_{x y}(t)\right\|^{2} & +\frac{1}{4} t^{\alpha}\left\|V_{y y}(t)\right\|^{2} \\
& +C t^{-3 \alpha}\left(\sup _{x \in \mathbb{R}} \int_{\mathbb{R}} V^{2} d y\right)^{2}\left\|V_{x}(t)\right\|^{2} \\
\leq \frac{1}{2} t^{\alpha}\left\|V_{x y}(t)\right\|^{2} & +\frac{1}{4} t^{\alpha}\left\|V_{y y}(t)\right\|^{2} \\
& +C t^{-3 \alpha}\left(\int_{\mathbb{R}}\left(\sup _{x \in \mathbb{R}} V\right)^{2} d y\right)^{2}\left\|V_{x}(t)\right\|^{2} \\
& +C t^{-3 \alpha}\left\|V_{x}(t)\right\|^{4}\|V(t)\|^{2} . \\
\leq \frac{1}{2} t^{\alpha}\left\|V_{x y}(t)\right\|^{2} & +\frac{1}{4} t^{\alpha}\left\|V_{y y}(t)\right\|^{2} \\
& +C t^{-3 \alpha}\left(\int_{\mathbb{R}}\left\|V_{x}(t)\right\|_{L^{2}\left(\mathbb{R}_{x}\right)}\|V(t)\|_{L^{2}\left(\mathbb{R}_{x}\right)} d y\right)^{2}\left\|V_{x}(t)\right\|^{2} \\
\leq \frac{1}{2} t^{\alpha}\left\|V_{x y}(t)\right\|^{2}+ & \frac{1}{4} t^{\alpha}\left\|V_{y y}(t)\right\|^{2} \\
&
\end{aligned}
$$

Similarly,

$$
I_{13} \leq \frac{1}{4} t^{\alpha}\left\|V_{y y}(t)\right\|^{2}+C t^{-3 \alpha}\|V(t)\|^{2}\left\|V_{x}(t)\right\|^{2}\left\|V_{y}(t)\right\|^{2} .
$$


Substituting (4.27) and (4.28) into (4.26), we have

$$
\begin{aligned}
& \frac{1}{2} \frac{d}{d t}\left\|V_{y}(t)\right\|^{2}+\frac{\beta}{2}\left\|\left(\sqrt{U_{x}} V_{y}\right)(t)\right\|^{2}+\frac{1}{2} t^{\alpha}\left\|\nabla V_{y}(t)\right\|^{2} \\
& \quad \leq C t^{-3 \alpha}(1+t)^{-1-\alpha}\left\|V_{x}(t)\right\|^{2}\left(\left\|V_{x}(t)\right\|^{2}+\left\|V_{y}(t)\right\|^{2}\right)\left(\left\|V_{0}\right\|_{L^{1}}^{2}+\left\|V_{0}\right\|_{1}^{2}+1\right) .
\end{aligned}
$$

Multiplying (4.29) by $(1+t)^{2+\alpha+\varepsilon}$, and integrating the resulting inequality over $[0, t]$ in $t$, we obtain (4.2) from (4.1) and (4.23).

Next, we will show the decay rate of $V_{x}$. Multiplying (2.16) by $-V_{x x}$, and integrating the resulting equation over $\mathbb{R}^{2}$, we have

$$
\begin{aligned}
& \frac{1}{2} \frac{d}{d t}\left\|V_{x}(t)\right\|^{2}-\int_{\mathbb{R}^{2}}(f(U+V)-f(U))_{x} V_{x x} d x d y-\int_{\mathbb{R}^{2}} g(U+V)_{y} V_{x x} d x d y \\
& \quad=-t^{\alpha} \int_{\mathbb{R}^{2}} V_{x x}^{2} d x d y-t^{\alpha} \int_{\mathbb{R}^{2}} V_{y y} V_{x x} d x d y .
\end{aligned}
$$

We estimate the second and third terms of the left-hand side of (4.30) respectively as follows:

$$
\begin{aligned}
-\int_{\mathbb{R}^{2}} & (f(U+V)-f(U))_{x} V_{x x} d x d y \\
= & -\int_{\mathbb{R}^{2}}\left(f^{\prime}(U+V)\left(U_{x}+V_{x}\right)-f^{\prime}(U) U_{x}\right) V_{x x} d x d y \\
= & -\int_{\mathbb{R}^{2}} f^{\prime}(U+V) V_{x} V_{x x} d x d y-\int_{\mathbb{R}^{2}}\left(f^{\prime}(U+V)-f^{\prime}(U)\right) U_{x} V_{x x} d x d y \\
= & \frac{1}{2} \int_{\mathbb{R}^{2}} f^{\prime \prime}(U+V)\left(U_{x}+V_{x}\right) V_{x}^{2} d x d y-\int_{\mathbb{R}^{2}} f^{\prime \prime}\left(\xi_{3}\right) V U_{x} V_{x x} d x d y
\end{aligned}
$$

where $\xi_{3}$ is defined by (4.15), and

$$
\begin{aligned}
& -\int_{\mathbb{R}^{2}} g(U+V)_{y} V_{x x} d x d y \\
& \quad=\int_{\mathbb{R}^{2}} g(U+V)_{y x} V_{x} d x d y \\
& =\int_{\mathbb{R}^{2}}\left(g^{\prime \prime}(U+V)\left(U_{x}+V_{x}\right) V_{y}+g^{\prime}(U+V) V_{x y}\right) V_{x} d x d y \\
& =\int_{\mathbb{R}^{2}} g^{\prime \prime}(U+V) U_{x} V_{x} V_{y} d x d y+\int_{\mathbb{R}^{2}} g^{\prime \prime}(U+V) V_{x}^{2} V_{y} d x d y \\
& \quad-\frac{1}{2} \int_{\mathbb{R}^{2}} g^{\prime \prime}(U+V) V_{y} V_{x}^{2} d x d y \\
& =\int_{\mathbb{R}^{2}} g^{\prime \prime}(U+V) U_{x} V_{x} V_{y} d x d y+\frac{1}{2} \int_{\mathbb{R}^{2}} g^{\prime \prime}(U+V) V_{y} V_{x}^{2} d x d y .
\end{aligned}
$$


From (4.31) and (4.32), by the Cauchy-Schwarz inequality, we can rewrite (4.30) as

$$
\begin{aligned}
\frac{1}{2} & \frac{d}{d t}\left\|V_{x}(t)\right\|^{2}+\frac{\beta}{2}\left\|\left(\sqrt{U_{x}} V_{x}\right)(t)\right\|^{2}+t^{\alpha}|| \nabla V_{x}(t) \|^{2} \\
\leq & C \int_{\mathbb{R}^{2}}\left|V_{x}^{3}\right| d x d y+C \int_{\mathbb{R}^{2}}\left|V_{x}^{2} V_{y}\right| d x d y+C \int_{\mathbb{R}^{2}}\left|V U_{x} V_{x x}\right| d x d y \\
& +C \int_{\mathbb{R}^{2}}\left|U_{x} V_{x} V_{y}\right| d x d y \\
\leq & C \int_{\mathbb{R}^{2}}\left|V_{x}^{3}\right| d x d y+C \int_{\mathbb{R}^{2}}\left|V_{x}^{2} V_{y}\right| d x d y+C \int_{\mathbb{R}^{2}}\left|V U_{x} V_{x x}\right| d x d y \\
& +C \int_{\mathbb{R}^{2}}\left|U_{x} V_{y}^{2}\right| d x d y+\frac{\beta}{4}\left\|\left(\sqrt{U_{x}} V_{x}\right)(t)\right\|^{2} \\
= & I_{14}+I_{15}+I_{16}+I_{17}+\frac{\beta}{4}\left\|\left(\sqrt{U_{x}} V_{x}\right)(t)\right\|^{2} .
\end{aligned}
$$

As in (4.28) and (4.27), we have

$$
I_{14} \leq \frac{1}{4} t^{\alpha}\left\|V_{x x}(t)\right\|^{2}+C t^{-3 \alpha}\|V(t)\|^{2}\left\|V_{x}(t)\right\|^{2}\left\|V_{y}(t)\right\|^{2}
$$

and

$$
I_{15} \leq \frac{1}{2} t^{\alpha}|| V_{x y}(t)\left\|^{2}+\frac{1}{4} t^{\alpha}\right\| V_{x x}(t)\left\|^{2}+C t^{-3 \alpha}\right\| V_{y}(t)\left\|^{4}\right\| V(t) \|^{2} .
$$

For $I_{16}$, by using the Cauchy-Schwarz inequality, we have from (4.17),

$$
\begin{aligned}
I_{16} & \leq \frac{1}{4} t^{\alpha}\left\|V_{x x}(t)\right\|^{2}+C t^{-\alpha}\left\|\left(U_{x} V\right)(t)\right\|^{2} \\
& \leq \frac{1}{4} t^{\alpha}\left\|V_{x x}(t)\right\|^{2}+C t^{-\alpha}\left\|U_{x}(t)\right\|_{L^{\infty}}\left\|\left(\sqrt{U_{x}} V\right)(t)\right\|^{2} \\
& \leq \frac{1}{4} t^{\alpha}\left\|V_{x x}(t)\right\|^{2}+C t^{-\alpha}(1+t)^{-\frac{7}{8}+\frac{\alpha}{2}}\left\|\left(\sqrt{U_{x}} V\right)(t)\right\|^{2} .
\end{aligned}
$$

For $I_{17}$, we have from (4.17) and (4.2),

$$
I_{17} \leq C|| U_{x}(t)\left\|_{L^{\infty}}\right\| V_{y}(t) \|^{2} \leq C(1+t)^{-\frac{23}{8}-\frac{\alpha}{2}}\left(\left\|V_{0}\right\|_{L^{1}}^{2}+\left\|V_{0}\right\|_{1}^{2}+1\right)^{2} .
$$


Substituting (4.34)-(4.37) into (4.33), we have from (4.1),

$$
\begin{aligned}
& \frac{1}{2} \frac{d}{d t}\left\|V_{x}(t)\right\|^{2}+\frac{\beta}{4}\left\|\left(\sqrt{U_{x}} V_{x}\right)(t)\right\|^{2}+\frac{1}{2} t^{\alpha}\left\|\nabla V_{x}(t)\right\|^{2} \\
& \leq C t^{-3 \alpha}\|V(t)\|^{2}\left\|V_{y}(t)\right\|^{2}\left(\left\|V_{x}(t)\right\|^{2}+\left\|V_{y}(t)\right\|^{2}\right) \\
&+C t^{-\alpha}(1+t)^{-\frac{7}{8}+\frac{\alpha}{2}}\left\|\left(\sqrt{U_{x}} V\right)(t)\right\|^{2} \\
&+C(1+t)^{-\frac{23}{8}-\frac{\alpha}{2}}\left(\left\|V_{0}\right\|_{L^{1}}^{2}+\left\|V_{0}\right\|_{1}^{2}+1\right)^{2} \\
& \leq C t^{-3 \alpha}(1+t)^{-1-\alpha}\left\|V_{y}(t)\right\|^{2}\left(\left\|V_{x}(t)\right\|^{2}\right. \\
&\left.+\left\|V_{y}(t)\right\|^{2}\right)\left(\left\|V_{0}\right\|_{L^{1}}^{2}+\left\|V_{0}\right\|_{1}^{2}+1\right) \\
&+C t^{-\alpha}(1+t)^{-\frac{7}{8}+\frac{\alpha}{2}}\left\|\left(\sqrt{U_{x}} V\right)(t)\right\|^{2} \\
&+C(1+t)^{-\frac{23}{8}-\frac{\alpha}{2}}\left(\left\|V_{0}\right\|_{L^{1}}^{2}+\left\|V_{0}\right\|_{1}^{2}+1\right)^{2} .
\end{aligned}
$$

Multiplying (4.38) by $(1+t)^{\frac{15}{8}+\frac{\alpha}{2}+\varepsilon}$ and integrating the resulting inequality over $[0, t]$ in $t$, we get (4.3) from (4.1), (4.2), (4.23) and (4.18).

Finally, we estimate the second derivatives of $V$.

First, differentiating (2.16) twice in $y$, and multiplying it by $V_{y y}$, then integrating the resulting equation over $\mathbb{R}^{2}$, we have

$$
\begin{aligned}
& \frac{1}{2} \frac{d}{d t}\left\|V_{y y}(t)\right\|^{2}+\int_{\mathbb{R}^{2}}(f(U+V)-f(U))_{x y y} V_{y y} d x d y+\int_{\mathbb{R}^{2}} g(U+V)_{y y y} V_{y y} d x d y \\
& \quad=t^{\alpha} \int_{\mathbb{R}^{2}} V_{x x y y} V_{y y} d x d y+t^{\alpha} \int_{\mathbb{R}^{2}} V_{y y y y} V_{y y} d x d y .
\end{aligned}
$$

Integrating by parts, we find that the second and the third terms of the left-hand side of (4.39) can be estimated as

$$
\begin{aligned}
\int_{\mathbb{R}^{2}}(f(U+V)-f(U))_{x y y} V_{y y} d x d y \\
=-\int_{\mathbb{R}^{2}}(f(U+V) \\
\quad-f(U))_{y y} V_{x y y} d x d y \\
=-\int_{\mathbb{R}^{2}}\left(f^{\prime \prime}(U+V) V_{y}^{2}+f^{\prime}(U+V) V_{y y}\right) V_{x y y} d x d y \\
=\frac{1}{2} \int_{\mathbb{R}^{2}} f^{\prime \prime}(U+V)\left(U_{x}+V_{x}\right) V_{y y}^{2} d x d y-\int_{\mathbb{R}^{2}} f^{\prime \prime}(U+V) V_{y}^{2} V_{x y y} d x d y
\end{aligned}
$$


and

$$
\begin{array}{rl}
\int_{\mathbb{R}^{2}} & g(U+V)_{y y y} V_{y y} d x d y \\
= & -\int_{\mathbb{R}^{2}} g(U+V)_{y y} V_{y y y} d x d y \\
= & -\int_{\mathbb{R}^{2}}\left(g^{\prime \prime}(U+V) V_{y}^{2}+g^{\prime}(U+V) V_{y y}\right) V_{y y y} d x d y \\
= & -\int_{\mathbb{R}^{2}} g^{\prime \prime}(U+V) V_{y}^{2} V_{y y y} d x d y+\frac{1}{2} \int_{\mathbb{R}^{2}} g^{\prime \prime}(U+V) V_{y} V_{y y}^{2} d x d y .
\end{array}
$$

From (4.40), (4.41), we rewrite (4.39) as

$$
\begin{aligned}
\frac{1}{2} \frac{d}{d t} \| & V_{y y}(t)\left\|^{2}+\frac{\beta}{2}\right\|\left(\sqrt{U_{x}} V_{y y}\right)(t)\left\|^{2}+t^{\alpha}|| \nabla V_{y y}(t)\right\|^{2} \\
\leq & C \int_{\mathbb{R}^{2}}\left|V_{x} V_{y y}^{2}\right| d x d y+C \int_{\mathbb{R}^{2}}\left|V_{y} V_{y y}^{2}\right| d x d y \\
& +C \int_{\mathbb{R}^{2}}\left|V_{y}^{2} V_{x y y}\right| d x d y+C \int_{\mathbb{R}^{2}}\left|V_{y}^{2} V_{y y y}\right| d x d y \\
= & I_{18}+I_{19}+I_{20}+I_{21} .
\end{aligned}
$$

We will respectively give the estimates of $I_{18}-I_{21}$. For $I_{18}$, by using the Sobolev inequality, the Gagliardo-Nirenberg inequality and the Cauchy-Schwarz inequality, we have

$$
\begin{aligned}
I_{18} \leq & C \int_{\mathbb{R}}\left\|V_{x}(t)\right\|_{L^{\infty}\left(\mathbb{R}_{y}\right)}\left\|V_{y y}(t)\right\|_{L^{2}\left(\mathbb{R}_{y}\right)}^{2} d x \\
\leq & C \int_{\mathbb{R}}\left\|V_{x y}(t)\right\|_{L^{2}\left(\mathbb{R}_{y}\right)}^{\frac{1}{2}}\left\|V_{x}(t)\right\|_{L^{2}\left(\mathbb{R}_{y}\right)}^{\frac{1}{2}}\left\|V_{y y}(t)\right\|_{L^{2}\left(\mathbb{R}_{y}\right)}^{2} d x \\
\leq & C t^{\alpha}(1+t)^{-1}\left\|V_{x y}(t)\right\|^{2}+C t^{-\frac{\alpha}{3}}(1+t)^{\frac{1}{3}} \int_{\mathbb{R}}\left\|V_{x}(t)\right\|_{L^{2}\left(\mathbb{R}_{y}\right)}^{\frac{2}{3}}\left\|V_{y y}(t)\right\|_{L^{2}\left(\mathbb{R}_{y}\right)}^{\frac{8}{3}} d x \\
\leq & C t^{\alpha}(1+t)^{-1}\left\|V_{x y}(t)\right\|^{2} \\
& \quad+C t^{-\frac{\alpha}{3}}(1+t)^{\frac{1}{3}} \int_{\mathbb{R}}\left\|V_{x}(t)\right\|_{L^{2}\left(\mathbb{R}_{y}\right)}^{\frac{2}{3}}\left\|V_{y y y}(t)\right\|_{L^{2}\left(\mathbb{R}_{y}\right)}^{\frac{4}{3}}\left\|V_{y}(t)\right\|_{L^{2}\left(\mathbb{R}_{y}\right)}^{\frac{4}{3}} d x \\
& \leq \frac{1}{8} t^{\alpha}\left\|V_{y y y}(t)\right\|^{2}+C t^{\alpha}(1+t)^{-1}\left\|V_{x y}(t)\right\|^{2} \\
& +C t^{-3 \alpha}(1+t)\left(\sup _{x}\left\|V_{y}(t)\right\|_{L^{2}\left(\mathbb{R}_{y}\right)}^{2}\right)^{2} \int_{\mathbb{R}}\left\|V_{x}(t)\right\|_{L^{2}\left(\mathbb{R}_{y}\right)}^{2} d x \\
\leq & \frac{1}{8} t^{\alpha}\left\|V_{y y y}(t)\right\|^{2}+C t^{\alpha}(1+t)^{-1}\left\|V_{x y}(t)\right\|^{2} \\
& \quad+C t^{-3 \alpha}(1+t)\left\|V_{x y}(t)\right\|^{2}\left\|V_{y}(t)\right\|^{2}\left\|V_{x}(t)\right\|^{2} .
\end{aligned}
$$


On the other hand, by using the Gagliardo-Nirenberg inequality, we have (4.44)

$$
\begin{aligned}
C t^{-3 \alpha}(1+t)\left\|V_{x y}(t)\right\|^{2}\left\|V_{y}(t)\right\|^{2}\left\|V_{x}(t)\right\|^{2} \\
\quad=C t^{-3 \alpha}(1+t)\left\|V_{y}(t)\right\|^{2}\left\|V_{x}(t)\right\|^{2} \int_{\mathbb{R}}\left\|V_{x y}(t)\right\|_{L^{2}\left(R_{y}\right)}^{2} d x \\
\quad \leq C t^{-3 \alpha}(1+t)\left\|V_{y}(t)\right\|^{2}\left\|V_{x}(t)\right\|^{2} \int_{\mathbb{R}}\left\|V_{x y y}(t)\right\|_{L^{2}\left(R_{y}\right)}\left\|V_{x}(t)\right\|_{L^{2}\left(R_{y}\right)} d x \\
\quad \leq C t^{-3 \alpha}(1+t)\left\|V_{x y y}(t)\right\|\left\|V_{x}(t)\right\|^{3}\left\|V_{y}(t)\right\|^{2} \\
\quad \leq \frac{1}{8} t^{\alpha}\left\|V_{x y y}(t)\right\|^{2}+C t^{-7 \alpha}(1+t)^{2}\left\|V_{x}(t)\right\|^{6}\left\|V_{y}(t)\right\|^{4} \\
\quad \leq \frac{1}{8} t^{\alpha}\left\|V_{x y y}(t)\right\|^{2}+C t^{-7 \alpha}(1+t)^{-\frac{61}{8}-\frac{7 \alpha}{2}}\left(\left\|V_{0}\right\|_{L^{1}}^{2}+\left\|V_{0}\right\|_{1}^{2}+1\right)^{10} .
\end{aligned}
$$

Therefore

$$
\begin{aligned}
I_{18} \leq & \frac{1}{8} t^{\alpha}\left\|V_{y y y}(t)\right\|^{2}+C t^{\alpha}(1+t)^{-1}\left\|V_{x y}(t)\right\|^{2} \\
& +\frac{1}{8} t^{\alpha}\left\|V_{x y y}(t)\right\|^{2}+C t^{-7 \alpha}(1+t)^{-\frac{61}{8}-\frac{7 \alpha}{2}}\left(\left\|V_{0}\right\|_{L^{1}}^{2}+\left\|V_{0}\right\|_{1}^{2}+1\right)^{10} .
\end{aligned}
$$

Similarly,

$$
\begin{aligned}
I_{19} \leq & \frac{1}{8} t^{\alpha}\left\|V_{y y y}(t)\right\|^{2}+C t^{\alpha}(1+t)^{-1}\left\|V_{y y}(t)\right\|^{2} \\
& +\frac{1}{8} t^{\alpha}\left\|V_{x y y}(t)\right\|^{2}+C t^{-7 \alpha}(1+t)^{-\frac{63}{8}-\frac{9 \alpha}{2}}\left(\left\|V_{0}\right\|_{L^{1}}^{2}+\left\|V_{0}\right\|_{1}^{2}+1\right)^{10} .
\end{aligned}
$$

For $I_{20}$, by using the Cauchy-Schwarz inequality and the Sobolev inequality, we get from (4.2),

$$
\begin{aligned}
I_{20} & \leq \frac{1}{32} t^{\alpha}\left\|V_{x y y}(t)\right\|^{2}+C t^{-\alpha}\left\|V_{y}(t)\right\|_{L^{4}}^{4} \\
& \leq \frac{1}{32} t^{\alpha}\left\|V_{x y y}(t)\right\|^{2}+C t^{-\alpha}\left\|V_{y}(t)\right\|_{L^{\infty}}^{2}\left\|V_{y}(t)\right\|^{2} \\
& \leq \frac{1}{32} t^{\alpha}\left\|V_{x y y}(t)\right\|^{2}+C t^{-\alpha}\left\|D^{2} V_{y}(t)\right\|\left\|V_{y}(t)\right\|^{3} \\
& \leq \frac{1}{16} t^{\alpha}\left\|D^{2} V_{y}(t)\right\|^{2}+C t^{-3 \alpha}\left\|V_{y}(t)\right\|^{6} \\
& \leq \frac{1}{16} t^{\alpha}\left\|D^{2} V_{y}(t)\right\|^{2}+C t^{-3 \alpha}(1+t)^{-6-3 \alpha}\left(\left\|V_{0}\right\|_{L^{1}}^{2}+\left\|V_{0}\right\|_{1}^{2}+1\right)^{6} .
\end{aligned}
$$

Similarly, we have

$$
I_{21} \leq \frac{1}{16} t^{\alpha}\left\|D^{2} V_{y}(t)\right\|^{2}+C t^{-3 \alpha}(1+t)^{-6-3 \alpha}\left(\left\|V_{0}\right\|_{L^{1}}^{2}+\left\|V_{0}\right\|_{1}^{2}+1\right)^{6} .
$$


Substituting (4.45)-(4.48) into (4.42), we arrive at

$$
\begin{aligned}
\frac{1}{2} \frac{d}{d t}\left\|V_{y y}(t)\right\|^{2}+\frac{\beta}{2}\left\|\left(\sqrt{U_{x}} V_{y y}\right)(t)\right\|^{2}+\frac{1}{4} t^{\alpha}\left\|\nabla V_{y y}(t)\right\|^{2} \\
\leq t^{\alpha}(1+t)^{-1}\left\|\nabla V_{y}(t)\right\|^{2}+C t^{-7 \alpha}(1+t)^{-\frac{61}{8}-\frac{7 \alpha}{2}}\left(\left\|V_{0}\right\|_{L^{1}}^{2}+\left\|V_{0}\right\|_{1}^{2}+1\right)^{10} \\
+C t^{-3 \alpha}(1+t)^{-6-3 \alpha}\left(\left\|V_{0}\right\|_{L^{1}}^{2}+\left\|V_{0}\right\|_{1}^{2}+1\right)^{6}+\frac{1}{8} t^{\alpha}\left\|V_{y x x}(t)\right\|^{2} .
\end{aligned}
$$

Multiplying $\frac{\partial^{2}(2.16)}{\partial x \partial y}$ by $V_{x y}$ and integrating the resulting equation over $\mathbb{R}^{2}$, we have

$$
\begin{aligned}
& \frac{1}{2} \frac{d}{d t}\left\|V_{x y}(t)\right\|^{2}+\int_{\mathbb{R}^{2}}(f(U+V)-f(U))_{x x y} V_{x y} d x d y+\int_{\mathbb{R}^{2}} g(U+V)_{y x y} V_{x y} d x d y \\
& \quad=t^{\alpha} \int_{\mathbb{R}^{2}} V_{x x x y} V_{x y} d x d y+t^{\alpha} \int_{\mathbb{R}^{2}} V_{y y x y} V_{x y} d x d y
\end{aligned}
$$

Integrating by parts, we find that the second and the third terms of the left-hand side of (4.50) can be estimated as

$$
\begin{aligned}
\int_{\mathbb{R}^{2}} & (f(U+V)-f(U))_{x x y} V_{x y} d x d y \\
= & -\int_{\mathbb{R}^{2}}(f(U+V)-f(U))_{x y} V_{x x y} d x d y \\
= & \frac{1}{2} \int_{\mathbb{R}^{2}} f^{\prime \prime}(U+V) U_{x} V_{x y}^{2} d x d y+\frac{1}{2} \int_{\mathbb{R}^{2}} f^{\prime \prime}(U+V) V_{x} V_{x y}^{2} d x d y \\
& -\int_{\mathbb{R}^{2}} f^{\prime \prime}(U+V) U_{x} V_{y} V_{x x y} d x d y-\int_{\mathbb{R}^{2}} f^{\prime \prime}(U+V) V_{x} V_{y} V_{x x y} d x d y
\end{aligned}
$$

and

$$
\begin{array}{rl}
\int_{\mathbb{R}^{2}} & g(U+V)_{y x y} V_{x y} d x d y \\
= & -\int_{\mathbb{R}^{2}} g^{\prime \prime}(U+V) V_{y}^{2} V_{x x y} d x d y-\int_{\mathbb{R}^{2}} g^{\prime}(U+V) V_{y y} V_{x x y} d x d y \\
= & -\int_{\mathbb{R}^{2}} g^{\prime \prime}(U+V) V_{y}^{2} V_{x x y} d x d y+\int_{\mathbb{R}^{2}} g^{\prime \prime}(U+V)\left(U_{x}+V_{x}\right) V_{y y} V_{x y} d x d y \\
& +\int_{\mathbb{R}^{2}} g^{\prime}(U+V) V_{x y y} V_{x y} d x d y \\
= & -\int_{\mathbb{R}^{2}} g^{\prime \prime}(U+V) V_{y}^{2} V_{x x y} d x d y+\int_{\mathbb{R}^{2}} g^{\prime \prime}(U+V) U_{x} V_{y y} V_{x y} d x d y \\
& +\int_{\mathbb{R}^{2}} g^{\prime \prime}(U+V) V_{x} V_{y y} V_{x y} d x d y-\frac{1}{2} \int_{\mathbb{R}^{2}} g^{\prime \prime}(U+V) V_{y} V_{x y}^{2} d x d y .
\end{array}
$$


From (4.51), (4.52), by using the Cauchy-Schwarz inequality we rewrite (4.50) as (4.53)

$$
\begin{aligned}
\frac{1}{2} \frac{d}{d t} \| & V_{x y}(t)\left\|^{2}+\frac{\beta}{4}\right\|\left(\sqrt{U_{x}} V_{x y}\right)(t) \|^{2}+\left.t^{\alpha}|| \nabla V_{x y}(t)\right|^{2} \\
\leq & C \int_{\mathbb{R}^{2}}\left|V_{x} V_{x y}^{2}\right| d x d y+C \int_{\mathbb{R}^{2}}\left|V_{y} V_{x y}^{2}\right| d x d y+C \int_{\mathbb{R}^{2}}\left|V_{x} V_{y} V_{x x y}\right| d x d y \\
& +C \int_{\mathbb{R}^{2}}\left|V_{y}^{2} V_{x x y}\right| d x d y+C \int_{\mathbb{R}^{2}}\left|U_{x} V_{y} V_{x x y}\right| d x d y \\
& +C \int_{\mathbb{R}^{2}}\left|U_{x} V_{y y} V_{x y}\right| d x d y+C \int_{\mathbb{R}^{2}}\left|V_{x} V_{y y} V_{x y}\right| d x d y \\
\leq & C \int_{\mathbb{R}^{2}}\left|V_{x} V_{x y}^{2}\right| d x d y+C \int_{\mathbb{R}^{2}}\left|V_{y} V_{x y}^{2}\right| d x d y+C \int_{\mathbb{R}^{2}}\left|V_{x} V_{y y}^{2}\right| d x d y \\
& +C \int_{\mathbb{R}^{2}}\left|V_{x}^{2} V_{x x y}\right| d x d y+C \int_{\mathbb{R}^{2}}\left|V_{y}^{2} V_{x x y}\right| d x d y \\
& +C \int_{\mathbb{R}^{2}}\left|U_{x} V_{y} V_{x x y}\right| d x d y+C \int_{\mathbb{R}^{2}}\left|U_{x} V_{y y}^{2}\right| d x d y \\
= & I_{22}+I_{23}+I_{24}+I_{25}+I_{26}+I_{27}+I_{28} .
\end{aligned}
$$

We will respectively give the estimates of $I_{22}-I_{28}$. Similar to the estimate of $I_{18}$, we have

$$
\begin{aligned}
I_{22} \leq & \frac{1}{16} t^{\alpha}\left\|V_{x x y}(t)\right\|^{2}+C t^{\alpha}(1+t)^{-1}\left\|V_{x x}(t)\right\|^{2} \\
& +\frac{1}{32} t^{\alpha}\left\|V_{y y y}(t)\right\|^{2}+C t^{-7 \alpha}(1+t)^{-\frac{31}{4}-4 \alpha}\left(\left\|V_{0}\right\|_{L^{1}}^{2}+\left\|V_{0}\right\|_{1}^{2}+1\right)^{10} .
\end{aligned}
$$

Continuing,

$$
\begin{aligned}
I_{23} \leq & \frac{1}{8} t^{\alpha}\left\|V_{y y x}(t)\right\|^{2}+C t^{\alpha}(1+t)^{-\frac{7}{8}+\frac{\alpha}{2}}\left\|V_{y y}(t)\right\|^{2} \\
& +\left.C t^{-3 \alpha}(1+t)^{\frac{7}{8}-\frac{\alpha}{2}}\left\|V_{x x}(t)\right\|^{2}\left\|V_{y}(t)\right\|\right|^{2}\left\|V_{x}(t)\right\|^{2} \\
\leq & \frac{1}{8} t^{\alpha}\left\|V_{y y x}(t)\right\|^{2}+C t^{\alpha}(1+t)^{-\frac{7}{8}+\frac{\alpha}{2}}\left\|V_{y y}(t)\right\|^{2} \\
& +\frac{1}{32} t^{\alpha}\left\|V_{x x x}(t)\right\|^{2}+C t^{-7 \alpha}(1+t)^{-\frac{63}{8}-\frac{9 \alpha}{2}}\left(\left\|V_{0}\right\|_{L^{1}}^{2}+\left\|V_{0}\right\|_{1}^{2}+1\right)^{10}
\end{aligned}
$$

and

$(4.56)$

$$
\begin{aligned}
I_{24} \leq & \frac{1}{8} t^{\alpha}\left\|V_{y y x}(t)\right\|^{2}+C t^{\alpha}(1+t)^{-\frac{7}{8}+\frac{\alpha}{2}}\left\|V_{x y}(t)\right\|^{2} \\
& +\frac{1}{32} t^{\alpha}\left\|V_{x x x}(t)\right\|^{2}+C t^{-7 \alpha}(1+t)^{-\frac{63}{8}-\frac{9 \alpha}{2}}\left(\left\|V_{0}\right\|_{L^{1}}^{2}+\left\|V_{0}\right\|_{1}^{2}+1\right)^{10} .
\end{aligned}
$$


For $I_{25}$, similar to the estimate of $I_{20}$, we have

$$
\begin{aligned}
I_{25} & \leq \frac{1}{32} t^{\alpha}\left\|V_{x x y}(t)\right\|^{2}+C t^{-\alpha}\left\|V_{x}(t)\right\|_{L^{4}}^{4} \\
& \leq \frac{1}{32} t^{\alpha}\left\|V_{x x y}(t)\right\|^{2}+C t^{-\alpha}\left\|D^{2} V_{x}(t)\right\|\left\|\mid V_{x}(t)\right\|^{3} \\
& \leq \frac{1}{16} t^{\alpha}\left\|D^{2} V_{x}(t)\right\|^{2}+C t^{-3 \alpha}(1+t)^{-\frac{45}{8}-\frac{3 \alpha}{2}}\left(\left\|V_{0}\right\|_{L^{1}}^{2}+\left\|V_{0}\right\|_{1}^{2}+1\right)^{6}
\end{aligned}
$$

and

$$
I_{26} \leq \frac{1}{16} t^{\alpha}\left\|D^{2} V_{y}(t)\right\|^{2}+C t^{-3 \alpha}(1+t)^{-6-3 \alpha}\left(\left\|V_{0}\right\|_{L^{1}}^{2}+\left\|V_{0}\right\|_{1}^{2}+1\right)^{6} .
$$

For $I_{27}$, according to (4.17) and the Cauchy-Schwarz inequality, we have

$$
\begin{aligned}
I_{27} & \leq \frac{1}{8} t^{\alpha}\left\|V_{x x y}(t)\right\|^{2}+C t^{-\alpha}\left\|U_{x}(t)\right\|_{L^{\infty}}^{2}\left\|V_{y}(t)\right\|^{2} \\
& \leq \frac{1}{8} t^{\alpha}\left\|V_{x x y}(t)\right\|^{2}+C t^{-\alpha}(1+t)^{-\frac{15}{4}}\left(\left\|V_{0}\right\|_{L^{1}}^{2}+\left\|V_{0}\right\|_{1}^{2}+1\right)^{2}
\end{aligned}
$$

and

$$
I_{28} \leq C|| U_{x}(t)\left\|_{L^{\infty}}\right\| V_{y y}(t)\left\|^{2} \leq C(1+t)^{-\frac{7}{8}+\frac{\alpha}{2}}\right\| V_{y y}(t) \|^{2} .
$$

Substituting (4.54)-(4.60) into (4.53), we have (4.61)

$$
\begin{aligned}
\frac{1}{2} \frac{d}{d t} & \left\|V_{x y}(t)\right\|^{2}+\frac{\beta}{4}\left\|\left(\sqrt{U_{x}} V_{x y}\right)(t)\right\|^{2}+\frac{1}{4} t^{\alpha}\left\|\nabla V_{x y}(t)\right\|^{2} \\
\leq & C t^{\alpha}(1+t)^{-1}\left\|V_{x x}(t)\right\|^{2}+C t^{-7 \alpha}(1+t)^{-\frac{31}{4}-4 \alpha}\left(\left\|V_{0}\right\|_{L^{1}}^{2}+\left\|V_{0}\right\|_{1}^{2}+1\right)^{10} \\
& +C t^{\alpha}(1+t)^{-\frac{7}{8}+\frac{\alpha}{2}}\left\|\nabla V_{y}(t)\right\|^{2}+C t^{-3 \alpha}(1+t)^{-\frac{45}{8}-\frac{3 \alpha}{2}}\left(\left\|V_{0}\right\|_{L^{1}}^{2}+\left\|V_{0}\right\|_{1}^{2}+1\right)^{6} \\
& +C t^{-\alpha}(1+t)^{-\frac{15}{4}}\left(\left\|V_{0}\right\|_{L^{1}}^{2}+\left\|V_{0}\right\|_{1}^{2}+1\right)^{2}+\frac{1}{8} t^{\alpha}\left\|V_{x x x}(t)\right\|^{2}+\frac{1}{16} t^{\alpha}\left\|V_{y y y}(t)\right\|^{2} .
\end{aligned}
$$

Multiplying $\frac{\partial^{2}(2.16)}{\partial x^{2}}$ by $V_{x x}(t)$ and integrating the resulting equation over $\mathbb{R}^{2}$, we have

$$
\begin{aligned}
& \frac{1}{2} \frac{d}{d t}\left\|V_{x x}(t)\right\|^{2}+\int_{\mathbb{R}^{2}}(f(U+V)-f(U))_{x x x} V_{x x} d x d y+\int_{\mathbb{R}^{2}} g(U+V)_{y x x} V_{x x} d x d y \\
& \quad=t^{\alpha} \int_{\mathbb{R}^{2}} V_{x x x x} V_{x x} d x d y+t^{\alpha} \int_{\mathbb{R}^{2}} V_{y y x x} V_{x x} d x d y .
\end{aligned}
$$


Integrating by parts, we find that the second term of the left-hand side of (4.65) can be estimated as

$$
\begin{aligned}
\int_{\mathbb{R}^{2}}( & f(U+V)-f(U))_{x x x} V_{x x} d x d y \\
=- & \int_{\mathbb{R}^{2}}(f(U+V)-f(U))_{x x} V_{x x x} d x d y \\
=- & \int_{\mathbb{R}^{2}} f^{\prime \prime}(U+V)\left(U_{x}+V_{x}\right)^{2} V_{x x x} d x d y \\
& -\int_{\mathbb{R}^{2}} f^{\prime}(U+V) U_{x x} V_{x x x} d x d y \\
& -\int_{\mathbb{R}^{2}} f^{\prime}(U+V) V_{x x} V_{x x x} d x d y \\
& +\int_{\mathbb{R}^{2}} f^{\prime \prime}(U) U_{x}^{2} V_{x x x} d x d y \\
= & \frac{1}{2} \int_{\mathbb{R}^{2}} f^{\prime \prime}(U+V)\left(U_{x}+V_{x}\right) V_{x x}^{2} d x d y \\
& -\int_{\mathbb{R}^{2}} f^{\prime \prime}\left(\xi_{3}\right) V U_{x x} V_{x x x} d x d y \\
- & \int_{\mathbb{R}^{2}} f^{\prime \prime \prime}\left(\xi_{4}\right) V U_{x}^{2} V_{x x x} d x d y-2 \int_{\mathbb{R}^{2}} f^{\prime \prime}(U+V) U_{x} V_{x} V_{x x x} d x d y \\
- & \int_{\mathbb{R}^{2}} f^{\prime \prime}(U+V) V_{x}^{2} V_{x x x} d x d y,
\end{aligned}
$$

where $\xi_{3}$ is defined by (4.15), and $\xi_{4}$ is between $U$ and $U+V$.

On the other hand,

$$
\begin{array}{rl}
\int_{\mathbb{R}^{2}} & g(U+V)_{y x x} V_{x x} d x d y \\
= & -\int_{\mathbb{R}^{2}}\left(g^{\prime \prime}(U+V)\left(U_{x}+V_{x}\right) V_{y}+g^{\prime}(U+V) V_{x y}\right) V_{x x x} d x d y \\
= & -\int_{\mathbb{R}^{2}} g^{\prime \prime}(U+V)\left(U_{x}+V_{x}\right) V_{y} V_{x x x} d x d y \\
& +\int_{\mathbb{R}^{2}} g^{\prime \prime}(U+V)\left(U_{x}+V_{x}\right) V_{x y} V_{x x} d x d y \\
& +\int_{\mathbb{R}^{2}} g^{\prime}(U+V) V_{x x y} V_{x x} d x d y \\
= & -\int_{\mathbb{R}^{2}} g^{\prime \prime}(U+V)\left(U_{x}+V_{x}\right) V_{y} V_{x x x} d x d y \\
& +\int_{\mathbb{R}^{2}} g^{\prime \prime}(U+V)\left(U_{x}+V_{x}\right) V_{x y} V_{x x} d x d y-\frac{1}{2} \int_{\mathbb{R}^{2}} g^{\prime \prime}(U+V) V_{y} V_{x x}^{2} d x d y .
\end{array}
$$


From (4.63) and (4.64), we rewrite (4.62) as (4.65)

$$
\begin{aligned}
\frac{1}{2} & \left.\frac{d}{d t}|| V_{x x}(t)\right|^{2}+\frac{\beta}{2} \|\left.\left(\sqrt{U_{x}} V_{x x}\right)(t)\right|^{2}+\left.t^{\alpha}|| \nabla V_{x x}(t)\right|^{2} \\
\leq & C \int_{\mathbb{R}^{2}}\left|V_{x} V_{x x}^{2}\right| d x d y+C \int_{\mathbb{R}^{2}}\left|V U_{x x} V_{x x x}\right| d x d y \\
& +C \int_{\mathbb{R}^{2}}\left|V U_{x}^{2} V_{x x x}\right| d x d y+C \int_{\mathbb{R}^{2}}\left|U_{x} V_{x} V_{x x x}\right| d x d y \\
& +C \int_{\mathbb{R}^{2}}\left|V_{x}^{2} V_{x x x}\right| d x d y+C \int_{\mathbb{R}^{2}}\left|U_{x} V_{y} V_{x x x}\right| d x d y+C \int_{\mathbb{R}^{2}}\left|V_{x} V_{y} V_{x x x}\right| d x d y \\
& +C \int_{\mathbb{R}^{2}}\left|U_{x} V_{x x} V_{x y}\right| d x d y+C \int_{\mathbb{R}^{2}}\left|V_{x} V_{x y} V_{x x}\right| d x d y+C \int_{\mathbb{R}^{2}}\left|V_{y} V_{x x}^{2}\right| d x d y \\
\leq & C \int_{\mathbb{R}^{2}}\left|V_{x} V_{x y}^{2}\right| d x d y+C \int_{\mathbb{R}^{2}}\left|V_{x} V_{x x}^{2}\right| d x d y+C \int_{\mathbb{R}^{2}}\left|V_{y} V_{x x}^{2}\right| d x d y \\
& +C \int_{\mathbb{R}^{2}}\left|V_{x}^{2} V_{x x x}\right| d x d y+C \int_{\mathbb{R}^{2}}\left|V_{y}^{2} V_{x x x}\right| d x d y+C \int_{\mathbb{R}^{2}}\left|V U_{x}^{2} V_{x x x}\right| d x d y \\
& +C \int_{\mathbb{R}^{2}}\left|U_{x} V_{y} V_{x x x}\right| d x d y+C \int_{\mathbb{R}^{2}}\left|V U_{x x} V_{x x x}\right| d x d y+C \int_{\mathbb{R}^{2}}\left|U_{x} V_{x y}^{2}\right| d x d y \\
= & I_{22}+I_{29}+I_{30}+I_{31}+I_{32}+I_{33}+I_{34}+I_{35}+I_{36},
\end{aligned}
$$

where $I_{22}$ is defined by (4.53).

Similar to $I_{18}$, we have $(4.66)$

$$
\begin{aligned}
I_{29} \leq & \frac{1}{16} t^{\alpha}\left\|V_{x x x}(t)\right\|^{2}+C t^{\alpha}(1+t)^{-1}\left\|V_{x x}(t)\right\|^{2} \\
& +\frac{1}{16} t^{\alpha}\left\|V_{x x y}(t)\right\|^{2}+C t^{-7 \alpha}(1+t)^{-\frac{15}{2}-3 \alpha}\left(\left\|V_{0}\right\|_{L^{1}}^{2}+\left\|V_{0}\right\|_{1}^{2}+1\right)^{10}
\end{aligned}
$$

and

(4.67)

$$
\begin{aligned}
I_{30} \leq & \frac{1}{16} t^{\alpha}|| V_{x x x}(t)\left\|^{2}+C t^{\alpha}(1+t)^{-1}\right\| V_{x y}(t) \|^{2} \\
& +\frac{1}{16} t^{\alpha}\left\|V_{x x y}(t)\right\|^{2}+C t^{-7 \alpha}(1+t)^{-\frac{31}{4}-4 \alpha}\left(\left\|V_{0}\right\|_{L^{1}}^{2}+\left\|V_{0}\right\|_{1}^{2}+1\right)^{10} .
\end{aligned}
$$

Similar to $I_{20}$, we have

$$
I_{31} \leq \frac{1}{16} t^{\alpha}\left\|D^{2} V_{x}(t)\right\|^{2}+C t^{-3 \alpha}(1+t)^{-\frac{45}{8}-\frac{3 \alpha}{2}}\left(\left\|V_{0}\right\|_{L^{1}}^{2}+\left\|V_{0}\right\|_{1}^{2}+1\right)^{6}
$$

and

(4.69)

$I_{32} \leq \frac{1}{16} t^{\alpha}\left\|V_{x x x}(t)\right\|^{2}+\frac{1}{32} t^{\alpha}\left\|D^{2} V_{y}(t)\right\|^{2}+C t^{-3 \alpha}(1+t)^{-6-3 \alpha}\left(\left\|V_{0}\right\|_{L^{1}}^{2}+\left\|V_{0}\right\|_{1}^{2}+1\right)^{6}$.

For $I_{33}$, by using the Cauchy-Schwarz inequality, we have from (4.17) and (4.1),

$$
\begin{aligned}
I_{33} & \leq \frac{1}{16} t^{\alpha}\left\|V_{x x x}(t)\right\|^{2}+C t^{-\alpha}\left\|U_{x}(t)\right\|_{L^{\infty}}^{4}\|V(t)\|^{2} \\
& \leq \frac{1}{16} t^{\alpha}\left\|V_{x x x}(t)\right\|^{2}+C t^{-\alpha}(1+t)^{-\frac{9}{2}+\alpha}\left(\left\|V_{0}\right\|_{L^{1}}^{2}+\left\|V_{0}\right\|^{2}+1\right) .
\end{aligned}
$$


Similarly,

$$
\begin{aligned}
I_{34} & \leq \frac{1}{16} t^{\alpha}\left\|V_{x x x}(t)\right\|^{2}+C t^{-\alpha}\left\|U_{x}(t)\right\|_{L^{\infty}}^{2}\left\|V_{y}(t)\right\|^{2} \\
& \leq \frac{1}{16} t^{\alpha}\left\|V_{x x x}(t)\right\|^{2}+C t^{-\alpha}(1+t)^{-\frac{15}{4}}\left(\left\|V_{0}\right\|_{L^{1}}^{2}+\left\|V_{0}\right\|_{1}^{2}+1\right)^{2} .
\end{aligned}
$$

Computing $I_{35}$ as above, we have

$$
I_{35} \leq \frac{1}{16} t^{\alpha}\left\|V_{x x x}(t)\right\|^{2}+C t^{-\alpha}\left\|U_{x x}(t)\right\|_{L^{\infty}}^{2}\|V(t)\|^{2} .
$$

Recalling that $U=w+v$, we have from (2.9) and (3.5),

$$
\begin{aligned}
\left\|U_{x x}(t)\right\|_{L^{\infty}}^{2} \leq & \left\|w_{x x}(t)\right\|_{L^{\infty}}^{2}+\left\|v_{x x}(t)\right\|_{L^{\infty}}^{2} \\
\leq & C(1+t)^{-3}+C\left\|v_{x x x}(t)\right\|\left\|v_{x x}(t)\right\| \\
\leq & +C(1+t)^{-3}+C t^{2 \alpha}(1+t)^{\frac{7}{16}-\frac{3 \alpha}{2}}\left\|v_{x x x}(t)\right\|^{2} \\
& +C t^{-2 \alpha}(1+t)^{-\frac{7}{16}+\frac{3 \alpha}{2}}\left\|v_{x x}(t)\right\|^{2} \\
\leq & +C(1+t)^{-3}+C t^{2 \alpha}(1+t)^{\frac{7}{16}-\frac{3 \alpha}{2}}\left\|v_{x x x}(t)\right\|^{2} \\
& +C t^{-2 \alpha}(1+t)^{-\frac{39}{16}+\frac{5 \alpha}{2}} .
\end{aligned}
$$

Therefore by (4.1)

$$
\begin{aligned}
I_{35} \leq & \frac{1}{16} t^{\alpha}\left\|V_{x x x}(t)\right\|^{2}+C t^{-\alpha}(1+t)^{-4-\alpha}\left(\left\|V_{0}\right\|_{L^{1}}^{2}+\left\|V_{0}\right\|^{2}+1\right) \\
& +C t^{\alpha}(1+t)^{-\frac{9}{16}-\frac{5 \alpha}{2}}\left\|v_{x x x}(t)\right\|^{2} \\
& +C t^{-3 \alpha}(1+t)^{-\frac{55}{16}+\frac{3 \alpha}{2}}\left(\left\|V_{0}\right\|_{L^{1}}^{2}+\left\|V_{0}\right\|^{2}+1\right) .
\end{aligned}
$$

For $I_{36}$, we have from (4.17) and (4.2),

$$
\begin{aligned}
I_{36} & \leq\left\|U_{x}(t)\right\|_{L^{\infty}}\left\|V_{x y}(t)\right\|^{2} \\
& \leq\left\|U_{x}(t)\right\| L_{L^{\infty}}\left\|V_{x x y}(t)\right\|\left\|V_{y}(t)\right\| \\
& \leq \frac{1}{16} t^{\alpha}\left\|V_{x x y}(t)\right\|^{2}+C t^{-\alpha}\left\|U_{x}(t)\right\|_{L^{\infty}}^{2}\left\|V_{y}(t)\right\|^{2} \\
& \leq \frac{1}{16} t^{\alpha}\left\|V_{x x y}(t)\right\|^{2}+C t^{-\alpha}(1+t)^{-\frac{15}{4}}\left(\left\|V_{0}\right\|_{L^{1}}^{2}+\left\|V_{0}\right\|_{1}^{2}+1\right)^{2} .
\end{aligned}
$$


Substituting (4.66)-(4.73) and (4.54) into (4.65), we have (4.74)

$$
\begin{aligned}
\frac{1}{2} \frac{d}{d t} \| & V_{x x}(t)\left\|^{2}+\frac{\beta}{4}\right\|\left(\sqrt{U_{x}} V_{x x}\right)(t)\left\|^{2}+\frac{1}{2} t^{\alpha}\right\| \nabla V_{x x}(t) \|^{2} \\
\leq & C t^{-3 \alpha}(1+t)^{-\frac{55}{16}+\frac{3 \alpha}{2}}\left(\left\|V_{0}\right\|_{L^{1}}^{2}+\left\|V_{0}\right\|_{1}^{2}+1\right)^{6} \\
& +C t^{-\alpha}(1+t)^{-\frac{15}{4}}\left(\left\|V_{0}\right\|_{L^{1}}^{2}+\left\|V_{0}\right\|_{1}^{2}+1\right)^{2} \\
& +C t^{-7 \alpha}(1+t)^{-\frac{15}{2}-3 \alpha}\left(\left\|V_{0}\right\|_{L^{1}}^{2}+\left\|V_{0}\right\|_{1}^{2}+1\right)^{10}+C t^{\alpha}(1+t)^{-1}\left\|\nabla V_{x}(t)\right\|^{2} \\
& +C t^{\alpha}(1+t)^{-\frac{9}{16}-\frac{5 \alpha}{2}}\left\|v_{x x x}(t)\right\|^{2}+\frac{1}{8} t^{\alpha}\left\|V_{x y y}(t)\right\|^{2}+\frac{1}{16} t^{\alpha}\left\|V_{y y y}(t)\right\|^{2} .
\end{aligned}
$$

Adding (4.49) and (4.61) to (4.74), we have

$$
\begin{aligned}
\frac{1}{2} \frac{d}{d t} & \left\|V_{y y}(t)\right\|^{2}+\frac{\beta}{2}\left\|\left(\sqrt{U_{x}} V_{y y}\right)(t)\right\|^{2}+\frac{1}{8} t^{\alpha}\left\|\nabla V_{y y}(t)\right\|^{2} \\
& +\frac{1}{2} \frac{d}{d t}\left\|V_{x y}(t)\right\|^{2}+\frac{\beta}{4}\left\|\left(\sqrt{U_{x}} V_{x y}\right)(t)\right\|^{2} \\
& +\frac{1}{8} t^{\alpha}\left\|\nabla V_{x y}(t)\right\|^{2} \\
& +\frac{1}{2} \frac{d}{d t}\left\|V_{x x}(t)\right\|^{2}+\frac{\beta}{2}\left\|\left(\sqrt{U_{x}} V_{x x}\right)(t)\right\|^{2} \\
& +\frac{1}{8} t^{\alpha}\left\|\nabla V_{x x}(t)\right\|^{2} \\
\leq & C t^{\alpha}(1+t)^{-1}\left\|\nabla V_{x}(t)\right\|^{2} \\
& +C t^{\alpha}(1+t)^{-\frac{7}{8}+\frac{\alpha}{2}}\left\|\nabla V_{y}(t)\right\|^{2} \\
& +C t^{-7 \alpha}(1+t)^{-\frac{15}{2}-3 \alpha}\left(\left\|V_{0}\right\|_{L^{1}}^{2}+\left\|V_{0}\right\|_{1}^{2}+1\right)^{10} \\
& +C t^{-3 \alpha}(1+t)^{-\frac{55}{16}+\frac{3 \alpha}{2}}\left(\left\|V_{0}\right\|_{L^{1}}^{2}+\left\|V_{0}\right\|_{1}^{2}+1\right)^{6} \\
& +C t^{-\alpha}(1+t)^{-\frac{15}{4}}\left(\left\|V_{0}\right\|_{L^{1}}^{2}+\left\|V_{0}\right\|_{1}^{2}+1\right)^{2} \\
& +C t^{\alpha}(1+t)^{-\frac{9}{16}-\frac{5 \alpha}{2}}\left\|v_{x x x}(t)\right\|^{2} .
\end{aligned}
$$

Integrating (4.75) over $[0, t]$ in $t$, we get from (4.23) and (3.34),

$$
\begin{aligned}
\left\|V_{y y}(t)\right\|^{2} & +\int_{0}^{t}\left(\left\|\left(\sqrt{U_{x}} V_{y y}\right)(\tau)\right\|^{2}+\tau^{\alpha}\left\|\nabla V_{y y}(\tau)\right\|^{2}\right) d \tau \\
& +\left\|V_{x y}(t)\right\|^{2}+\int_{0}^{t}\left(\left\|\left(\sqrt{U_{x}} V_{x y}\right)(\tau)\right\|^{2}+\tau^{\alpha}\left\|\nabla V_{x y}(\tau)\right\|^{2}\right) d \tau \\
& +\left\|V_{x x}(t)\right\|^{2}+\int_{0}^{t}\left(\left\|\left(\sqrt{U_{x}} V_{x x}\right)(\tau)\right\|^{2}+\tau^{\alpha}\left\|\nabla V_{x x}(\tau)\right\|^{2}\right) d \tau \\
& \leq C\left(\left\|V_{0}\right\|_{L^{1}}^{2}+\left\|V_{0}\right\|_{2}^{2}+1\right)^{10} .
\end{aligned}
$$


After obtaining the estimate (4.76), we can study the decay rate of $D^{2} V$. Multiplying $(4.75)$ by $(1+t)^{\frac{39}{16}+\frac{3 \alpha}{2}+\varepsilon}$, and integrating the resulting inequality over $[0, t]$ in $t$, we get from (4.2), (4.3), (3.5) and (4.76),

$$
\begin{aligned}
&(1+t)^{\frac{39}{16}+\frac{3 \alpha}{2}+\varepsilon}\left\|V_{y y}(t)\right\|^{2} \\
&+\int_{0}^{t}(1+\tau)^{\frac{39}{16}+\frac{3 \alpha}{2}+\varepsilon}\left(\left\|\left(\sqrt{U_{x}} V_{y y}\right)(\tau)\right\|^{2}+\tau^{\alpha}\left\|\nabla V_{y y}(\tau)\right\|^{2}\right) d \tau \\
&+(1+t)^{\frac{39}{16}+\frac{3 \alpha}{2}+\varepsilon}\left\|V_{x y}(t)\right\|^{2} \\
&+\int_{0}^{t}(1+\tau)^{\frac{39}{16}+\frac{3 \alpha}{2}+\varepsilon}\left(\left\|\left(\sqrt{U_{x}} V_{x y}\right)(\tau)\right\|^{2}+\tau^{\alpha}\left\|\nabla V_{x y}(\tau)\right\|^{2}\right) d \tau \\
&+(1+t)^{\frac{39}{16}+\frac{3 \alpha}{2}+\varepsilon}\left\|V_{x x}(t)\right\|^{2} \\
&+\int_{0}^{t}(1+\tau)^{\frac{39}{16}+\frac{3 \alpha}{2}+\varepsilon}\left(\left\|\left(\sqrt{U_{x}} V_{x x}\right)(\tau)\right\|^{2}+\tau^{\alpha}\left\|\nabla V_{x x}(\tau)\right\|^{2}\right) d \tau \\
& \leq\left\|V_{0 y y}\right\|^{2}+\left\|V_{0 x y}\right\|^{2}+\left\|V_{0 x x}\right\|^{2}+\int_{0}^{t} \tau^{\alpha}(1+\tau)^{\frac{23}{16}+\frac{3 \alpha}{2}+\varepsilon}\left\|\nabla V_{x}(\tau)\right\|^{2} d \tau \\
&+\int_{0}^{t} \tau^{\alpha}(1+\tau)^{\frac{25}{16}+2 \alpha+\varepsilon}\left\|\nabla V_{y}(\tau)\right\|^{2} d \tau+\int_{0}^{t} \tau^{\alpha}(1+\tau)^{\frac{15}{8}-\alpha+\varepsilon}\left\|v_{x x x}(\tau)\right\|^{2} d \tau \\
&+C\left(\left\|V_{0}\right\|_{L^{1}}^{2}+\left\|V_{0}\right\|_{1}^{2}+1\right)^{10} \int_{0}^{t} \tau^{-7 \alpha}(1+\tau)^{-\frac{81}{16}-\frac{3 \alpha}{2}+\varepsilon} d \tau \\
&+C\left(\left\|V_{0}\right\|_{L^{1}}^{2}+\left\|V_{0}\right\|_{1}^{2}+1\right)^{6} \int_{0}^{t} \tau^{-3 \alpha}(1+\tau)^{-1+3 \alpha+\varepsilon} d \tau \\
&+C\left(\left\|V_{0}\right\|_{L^{1}}^{2}+\left\|V_{0}\right\|_{1}^{2}+1\right)^{2} \int_{0}^{t} \tau^{-\alpha}(1+\tau)^{-\frac{21}{16}+\frac{3 \alpha}{2}+\varepsilon} d \tau \\
&+C \int_{0}^{t}(1+\tau)^{\frac{23}{16}+\frac{3 \alpha}{2}+\varepsilon}\left(\left\|V_{y y}(\tau)\right\|^{2}+\left\|V_{x y}(\tau)\right\|^{2}+\left\|V_{x x}(\tau)\right\|^{2}\right) d \tau \\
& \leq C(1+t)^{\varepsilon}\left(\left\|V_{0}\right\|_{L^{1}}^{2}+\left\|V_{0}\right\|_{2}^{2}+1\right)^{10} .
\end{aligned}
$$

This proves (4.6).

Now we turn to show (4.4) and (4.5). In order to obtain (4.4), we use another method to estimate $I_{20}$ and $I_{21}$. For $I_{20}$, we have from (4.47) and (4.2), (4.78)

$$
\begin{aligned}
I_{20} \leq & \frac{1}{32} t^{\alpha}\left\|V_{x y y}(t)\right\|^{2}+C t^{-\alpha}\left(\left\|V_{y y y}(t)\right\|+\left\|V_{y y x}(t)\right\|+\left\|V_{y x x}(t)\right\|\right)\left\|V_{y}(t)\right\|^{3} \\
\leq & \frac{1}{16} t^{\alpha}\left\|\nabla V_{y y}(t)\right\|^{2}+t^{\alpha}(1+t)^{-\frac{9}{16}+\frac{\alpha}{2}}\left\|V_{y x x}(t)\right\|^{2}+C t^{-3 \alpha}(1+t)^{\frac{9}{16}-\frac{\alpha}{2}}\left\|V_{y}(t)\right\|^{6} \\
\leq & \frac{1}{16} t^{\alpha}\left\|\nabla V_{y y}(t)\right\|^{2}+t^{\alpha}(1+t)^{-\frac{9}{16}+\frac{\alpha}{2}}\left\|V_{y x x}(t)\right\|^{2} \\
& +C t^{-3 \alpha}(1+t)^{-\frac{87}{16}-\frac{7 \alpha}{2}}\left(\left\|V_{0}\right\|_{L^{1}}^{2}+\left\|V_{0}\right\|_{1}^{2}+1\right)^{6} .
\end{aligned}
$$


Similarly,

$$
\begin{aligned}
I_{21} \leq & \frac{1}{16} t^{\alpha}\left\|\nabla V_{y y}(t)\right\|^{2}+t^{\alpha}(1+t)^{-\frac{9}{16}+\frac{\alpha}{2}}\left\|V_{y x x}(t)\right\|^{2} \\
& +C t^{-3 \alpha}(1+t)^{-\frac{87}{16}-\frac{7 \alpha}{2}}\left(\left\|V_{0}\right\|_{L^{1}}^{2}+\left\|V_{0}\right\|_{1}^{2}+1\right)^{6} .
\end{aligned}
$$

Substituting (4.45), (4.46), (4.78) and (4.79) into (4.42), we have

$$
\begin{aligned}
\frac{1}{2} \frac{d}{d t} & \left\|V_{y y}(t)\right\|^{2}+\frac{\beta}{2}\left\|\left(\sqrt{U_{x}} V_{y y}\right)(t)\right\|^{2}+\frac{1}{2} t^{\alpha}\left\|\nabla V_{y y}(t)\right\|^{2} \\
\leq & C t^{\alpha}(1+t)^{-1}\left\|\nabla V_{y}(t)\right\|^{2}+C t^{\alpha}(1+t)^{-\frac{9}{16}+\frac{\alpha}{2}}\left\|V_{y x x}(t)\right\|^{2} \\
& +C t^{-7 \alpha}(1+t)^{-\frac{61}{8}-\frac{7 \alpha}{2}}\left(\left\|V_{0}\right\|_{L^{1}}^{2}+\left\|V_{0}\right\|_{1}^{2}+1\right)^{10} \\
& +C t^{-3 \alpha}(1+t)^{-\frac{87}{16}-\frac{7 \alpha}{2}}\left(\left\|V_{0}\right\|_{L^{1}}^{2}+\left\|V_{0}\right\|_{1}^{2}+1\right)^{6} .
\end{aligned}
$$

Multiplying (4.80) by $(1+t)^{3+\alpha+\varepsilon}$, then integrating it over $[0, t]$ in $t$, we get from (4.76), (4.2) and (4.6),

$$
\begin{aligned}
& (1+t)^{3+\alpha+\varepsilon}\left\|V_{y y}(t)\right\|^{2}+\int_{0}^{t}(1+\tau)^{3+\alpha+\varepsilon}\left(\left\|\left(\sqrt{U_{x}} V_{y y}\right)(\tau)\right\|^{2}+\tau^{\alpha}\left\|\nabla V_{y y}(\tau)\right\|^{2}\right) d \tau \\
& \leq C\left\|V_{0 y y}\right\|^{2}+C \int_{0}^{t}(1+\tau)^{2+\alpha+\varepsilon}\left\|V_{y y}(\tau)\right\|^{2} d \tau \\
& \quad+C \int_{0}^{t} \tau^{\alpha}(1+\tau)^{2+\alpha+\varepsilon}\left\|\nabla V_{y}(\tau)\right\|^{2} d \tau+C \int_{0}^{t} \tau^{\alpha}(1+\tau)^{\frac{39}{16}+\frac{3 \alpha}{2}+\varepsilon}\left\|V_{y x x}(\tau)\right\|^{2} d \tau \\
& \quad+C\left(\left\|V_{0}\right\|_{L^{1}}^{2}+\left\|V_{0}\right\|_{1}^{2}+1\right)^{10} \int_{0}^{t} \tau^{-7 \alpha}(1+\tau)^{-\frac{37}{8}-\frac{5 \alpha}{2}+\varepsilon} d \tau \\
& \quad+C\left(\left\|V_{0}\right\|_{L^{1}}^{2}+\left\|V_{0}\right\|_{1}^{2}+1\right)^{6} \int_{0}^{t} \tau^{-3 \alpha}(1+\tau)^{-\frac{39}{16}-\frac{5 \alpha}{2}+\varepsilon} d \tau \\
& \leq C(1+t)^{\varepsilon}\left(\left\|V_{0}\right\|_{L^{1}}^{2}+\left\|V_{0}\right\|_{2}^{2}+1\right)^{10},
\end{aligned}
$$

which implies (4.4).

From (4.6), (4.4), we have

$$
\left\|V_{x x}(t)\right\|^{2} \leq C(1+t)^{-\frac{39}{16}-\frac{3 \alpha}{2}}\left(\left\|V_{0}\right\|_{L^{1}}^{2}+\left\|V_{0}\right\|_{2}^{2}+1\right)^{10}
$$

and

$$
\left\|V_{y y}(t)\right\|^{2} \leq C(1+t)^{-3-\alpha}\left(\left\|V_{0}\right\|_{L^{1}}^{2}+\left\|V_{0}\right\|_{2}^{2}+1\right)^{10} .
$$

Substituting (4.81) and (4.82) into (4.55), we have from (4.2) and (4.3),

$$
\begin{aligned}
I_{23} \leq & \frac{1}{8}\left\|V_{y y x}(t)\right\|^{2}+C t^{\alpha}(1+t)^{-\frac{31}{8}-\frac{\alpha}{2}}\left(\left\|V_{0}\right\|_{L^{1}}^{2}+\left\|V_{0}\right\|_{2}^{2}+1\right)^{10} \\
& +C t^{-3 \alpha}(1+t)^{-\frac{87}{16}-\frac{7 \alpha}{2}}\left(\left\|V_{0}\right\|_{L^{1}}^{2}+\left\|V_{0}\right\|_{2}^{2}+1\right)^{14} .
\end{aligned}
$$


As in (4.78), we use another method to estimate $I_{25}$ and $I_{27}$ as follows:

$$
\begin{gathered}
I_{25} \leq \frac{1}{8} t^{\alpha}\left\|V_{x x y}(t)\right\|^{2}+C t^{-\alpha}\left(\left\|V_{x x x}(t)\right\|+\left\|V_{x y y}(t)\right\|+\left\|V_{x x y}(t)\right\|\right)\left\|V_{x}(t)\right\|^{3} \\
\leq \leq \frac{1}{4} t^{\alpha}\left\|\nabla V_{x y}(t)\right\|^{2}+t^{\alpha}(1+t)^{-\frac{7}{16}+\alpha}\left\|V_{x x x}(t)\right\|^{2}+C t^{-3 \alpha}(1+t)^{\frac{7}{16}-\alpha}\left\|V_{x}(t)\right\|^{6} \\
\leq \frac{1}{4} t^{\alpha}\left\|\nabla V_{x y}(t)\right\|^{2}+t^{\alpha}(1+t)^{-\frac{7}{16}+\alpha}\left\|V_{x x x}(t)\right\|^{2} \\
+C t^{-3 \alpha}(1+t)^{-\frac{83}{16}-\frac{5 \alpha}{2}}\left(\left\|V_{0}\right\|_{L^{1}}^{2}+\left\|V_{0}\right\|_{1}^{2}+1\right)^{6}, \\
I_{27} \leq \frac{1}{8} t^{\alpha}|| V_{x x y}(t)\left\|^{2}+C t^{-\alpha}\right\| U_{x}(t)\left\|_{L^{\infty}}\right\|\left(\sqrt{U_{x}} V_{y}\right)(t) \|^{2} \\
\leq \frac{1}{8} t^{\alpha}\left\|V_{x x y}(t)\right\|^{2}+C t^{-\alpha}(1+t)^{-\frac{7}{8}+\frac{\alpha}{2}}\left\|\left(\sqrt{U_{x}} V_{y}\right)(t)\right\|^{2} .
\end{gathered}
$$

Substituting (4.83), (4.84), (4.85), (4.60), (4.54), (4.56) and (4.58) into (4.53), we have

(4.86)

$$
\begin{aligned}
& \frac{1}{2} \frac{d}{d t}\left\|V_{x y}(t)\right\|^{2}+\frac{\beta}{4}\left\|\left(\sqrt{U_{x}} V_{x y}\right)(t)\right\|^{2}+t^{\alpha}\left\|\nabla V_{x y}(t)\right\|^{2} \\
& \leq t^{\alpha}(1+t)^{-\frac{7}{8}+\frac{\alpha}{2}}\left\|\nabla V_{y}(t)\right\|^{2}+t^{\alpha}(1+t)^{-1}\left\|V_{x x}(t)\right\|^{2}+t^{\alpha}(1+t)^{-\frac{7}{16}+\alpha}\left\|V_{x x x}(t)\right\|^{2} \\
& \quad+C t^{-7 \alpha}(1+t)^{-\frac{31}{4}-4 \alpha}\left(\left\|V_{0}\right\|_{L^{1}}^{2}+\left\|V_{0}\right\|_{1}^{2}+1\right)^{10} \\
& \quad+C t^{-3 \alpha}(1+t)^{-\frac{83}{16}-\frac{5 \alpha}{2}}\left(\left\|V_{0}\right\|_{L^{1}}^{2}+\left\|V_{0}\right\|_{1}^{2}+1\right)^{6} \\
& \quad+C t^{-\alpha}(1+t)^{-\frac{7}{8}+\frac{\alpha}{2}}\left\|\left(\sqrt{U_{x}} V_{y}\right)(t)\right\|^{2}+\frac{1}{4} t^{\alpha}\left\|V_{y y y}(t)\right\|^{2} .
\end{aligned}
$$

Multiplying (4.86) by $(1+t)^{\frac{23}{8}+\frac{\alpha}{2}+\varepsilon}$, we get from (4.2), (4.3) and (4.4),

$$
\begin{aligned}
& (1+t)^{\frac{23}{8}+\frac{\alpha}{2}+\varepsilon}\left\|V_{x y}(t)\right\|^{2} \\
& \quad+\int_{0}^{t}(1+\tau)^{\frac{23}{8}+\frac{\alpha}{2}+\varepsilon}\left(\left\|\left(\sqrt{U_{x}} V_{y y}\right)(\tau)\right\|^{2}+\tau^{\alpha}\left\|\nabla V_{y y}(\tau)\right\|^{2}\right) d \tau \\
& \leq C\left\|V_{0 x y}\right\|^{2}+\int_{0}^{t}(1+\tau)^{\frac{15}{8}+\frac{\alpha}{2}+\varepsilon}\left\|V_{x y}(\tau)\right\|^{2} d \tau+\int_{0}^{t} \tau^{\alpha}(1+\tau)^{\frac{15}{8}+\frac{\alpha}{2}+\varepsilon}\left\|V_{x x}(\tau)\right\|^{2} d \tau \\
& \quad+\int_{0}^{t} \tau^{\alpha}(1+\tau)^{2+\alpha+\varepsilon}\left\|\nabla V_{y}(\tau)\right\|^{2} d \tau+C \int_{0}^{t} \tau^{\alpha}(1+\tau)^{\frac{15}{8}+\alpha+\varepsilon}\left\|\left(\sqrt{U_{x}} V_{y}\right)(\tau)\right\|^{2} d \tau \\
& \quad+\int_{0}^{t} \tau^{\alpha}(1+\tau)^{\frac{23}{8}+\frac{\alpha}{2}+\varepsilon}\left\|V_{y y y}(\tau)\right\|^{2} d \tau+\int_{0}^{t} \tau^{\alpha}(1+\tau)^{\frac{39}{16}+\frac{3 \alpha}{2}+\varepsilon}\left\|V_{x x x}(\tau)\right\|^{2} d \tau \\
& \quad+C\left(\left\|V_{0}\right\|_{L^{1}}^{2}+\left\|V_{0}\right\|_{1}^{2}+1\right)^{10} \int_{0}^{t} \tau^{-7 \alpha}(1+\tau)^{-\frac{39}{8}-\frac{7 \alpha}{2}+\varepsilon} d \tau \\
& \quad+C\left(\left\|V_{0}\right\|_{L^{1}}^{2}+\left\|V_{0}\right\|_{1}^{2}+1\right)^{14} \int_{0}^{t} \tau^{-3 \alpha}(1+\tau)^{-\frac{37}{16}-2 \alpha+\varepsilon} d \tau \\
& \leq C(1+t)^{\varepsilon}\left(\left\|V_{0}\right\|_{L^{1}}^{2}+\left\|V_{0}\right\|_{2}^{2}+1\right)^{14},
\end{aligned}
$$


which implies (4.5).

The proof of Theorem 4.2 is completed.

Finally, Theorem 1.1 follows from (3.3), (4.7) and (2.11).

\section{ACKNOWLEDGEMENT}

The authors would like to thank the anonymous referee for his/her helpful suggestions and comments. The research was supported by the National Natural Science Foundation of China \#10625105 and \#10431060, and the Program for New Century Excellent Talents in University \#NCET-04-0745.

\section{REFERENCES}

[1] D. G. Crighton, Model equation of nonlinear acoustics, Annual Rev. Fluid Mech., 11(1979), 11-33.

[2] D. G. Crighton and J. F. Scott, Asymptotic solutions of model equations in nonlinear acoustics, Philos. Trans. R. Soc. London, 292(A)(1979), 101-134. MR547939 (80h:76040)

[3] E. Harabetian, Rarefactions and large time behavior for parabolic equations and monotone schemes, Comm. Math. Phys., 114(1988), 527-536. MR929127 (89d:35084)

[4] Y. Hattori and K. Nishihara, A note on the stability of the rarefaction wave of the Burgers equation, Japan J. Indust. Appl. Math., 8(1991), 85-86. MR.1093830 (91k:35227)

[5] A. M. Il'in and O. A. Oleinik, Asymptotic behavior of solutions of the Cauchy problem for certain quasilinear equations for large time (Russian), Mat. Sb., 51(1960), 191-216. MR0120469 $(22: 11222)$

[6] K. Ito, Asymptotic decay toward the planar rarefaction waves of solutions for viscous conservation laws in several dimensions, Math. Models Methods Appl. Sci., 6(1996), 315-338. MR.1388709 (97e:35106)

[7] S. Kawashima and A. Matsumura, Asymptotic stability of travelling wave solutions of systems for one-dimensional gas motion, Comm. Math. Phys., 101(1985), 97-127. MR814544 (87h:35035)

[8] S. Leibovich and A. R. Seebass, eds., Nonlinear Waves, Cornell University Press, Ithaca, NY, 1974. MR0355283(50:7759)

[9] A. Matsumara and K. Nishihara, Global stability of the rarefaction wave of a one-dimensional model system for compressible viscious gas, Comm. Math. Phys., 144(1992), 325-335. MR1152375 (93d:76056)

[10] K. Nishihara, Asymptotic behaviors of solutions to viscous conservation laws via the $L^{2}-$ energy method, Lecture notes delivered in Summer School in Fudan University, Shanghai, China, 1999.

[11] M. Nishikawa and K. Nishihara, Asymptotics toward the planar rarefaction wave for viscous conservation law in two space dimensions, Trans. Amer. Math. Soc., 352(2000), 1203-1215 MR1491872 (2000j:35180)

[12] P. L. Sachdev, Nonlinear Diffusive Waves, Cambridge University Press, Cambridge, 1987. MR898438 (88g:35177)

[13] J. F. Scott, The long time asymptotics of solutions to the generalized Burgers equation, Proc. Roy. Soc. London, Ser. A, 373(1981), 443-456. MR603065 (82b:76052)

[14] J. H. Wang and H. Zhang, Existence and decay rates of smooth solutions for a non-uniformly parabolic equation, Proc. Roy. Soc. Edinburgh, Sect. A, 132(2002), 1477-1491. MR 1950818 (2003j:35192)

[15] Z. P. Xin, Asymptotic stability of planar rarefaction waves for viscous conservation laws in several dimensions, Trans. Amer. Math. Soc., 319(1990), 805-829. MR970270 (90j:35138)

[16] Y. L. Xu and M. N. Jiang, Asymptotic stability of rarefaction wave for generalized Burgers equation, Acta Math. Sci., 25(B)(2005), 119-129. MR2119344(2005j:35202)

[17] H. Zhang, Existence of weak solutions for a degenerate generalized Burgers equation with large initial data, Acta. Math. Sci, 22(B)(2002), 241-248. MR.1901484 (2003f:35179)

[18] H. J. Zhao, Nonlinear stability of strong planar rarefaction waves for the relaxation approximation of conservation laws in several space dimensions, J. Differential Equations, 163(2000), 198-222. MR.1755074 (2001c:35148) 
[19] C. J. Zhu, Asymptotic behavior of solutions for $p$-system with relaxation, J. Differential Equations, 180(2002), 273-306. MR1894014 (2003d:35174)

[20] C. J. Zhu and Z. A. Wang, Decay rates of solutions to dissipative nonlinear evolution equations with ellipticity, Z. Angew. Math. Phys., 55(2004), 1-21. MR2100527 (2005i:35114)

Department of Mathematics, Laboratory of Nonlinear Analysis, Central China Normal University, Wuhan 430079, People's Republic of China

Department of Mathematics, Laboratory of Nonlinear Analysis, Central China Normal University, Wuhan 430079, People's Republic of China

E-mail address: cjzhu@mail.ccnu.edu.cn 